\title{
Homologs of circadian clock proteins impact the metabolic switch between light and dark growth in the cyanobacterium Synechocystis sp. PCC 6803
}

\author{
Nina M. Scheurer ${ }^{1 \dagger}$, Yogeswari Rajarathinam ${ }^{2 \dagger}$, Stefan Timm $^{3}$, Christin Köbler1, Joachim \\ Kopka $^{2}$, Martin Hagemann ${ }^{3}$, Annegret Wilde ${ }^{1^{*}}$ \\ ${ }^{1}$ Institute of Biology III, University of Freiburg, Freiburg, Germany; \\ ${ }^{2}$ Applied Metabolome Analysis, Department of Molecular Physiology, Max Planck Institute of \\ Molecular Plant Physiology, Potsdam, Germany; \\ ${ }^{3}$ Department of Plant Physiology, University of Rostock, Rostock, Germany \\ †Shared co-first authorship \\ * Correspondence: \\ Annegret Wilde \\ Annegret.wilde@biologie.uni-freiburg.de
}

Keywords: circadian clock, Synechocystis, carbon metabolism, RpaA, diurnal metabolic switch

\begin{abstract}
The putative circadian clock system of the facultative heterotrophic cyanobacterial strain Synechocystis sp. PCC 6803 comprises the following three Kai-based systems: a KaiABC-based potential oscillator that is linked to the SasA-RpaA two-component output pathway and two additional KaiBC systems without a cognate KaiA component. Mutants lacking the genes encoding the KaiAB1C1 components or the response regulator RpaA show reduced growth in light/dark cycles and do not show heterotrophic growth in the dark. In the present study, the effect of these mutations on central metabolism was analyzed by targeted and nontargeted metabolite profiling. The strongest metabolic changes were observed in the dark in $\triangle r p a A$ and, to a lesser extent, in the $\triangle k a i A B 1 C l$ mutant. These observations included the overaccumulation of 2-phosphoglycolate, which correlated with the overaccumulation of the RbcL subunit in the mutants, and taken together, these data suggest enhanced RubisCO activity in the dark. The imbalanced carbon metabolism in the $\Delta r p a A$ mutant extended to the pyruvate family of amino acids, which showed increased accumulation in the dark. Hence, the deletion of the response regulator rpaA had a more pronounced effect on metabolism than the deletion of the kai genes. The larger impact of the rpaA mutation is in agreement with previous transcriptomic analyses and likely relates to a KaiAB1C1-independent function as a transcription factor. Collectively, our data demonstrate an important role of homologs of clock proteins in Synechocystis for balanced carbon and nitrogen metabolism during light-to-dark transitions.
\end{abstract}

\section{$1 \quad$ Introduction}

Most organisms on Earth experience daily environmental changes. Similar to eukaryotic organisms, photosynthetic cyanobacteria evolved a circadian clock system to respond to predictable fluctuations of light (L) and darkness (D) in a day-night cycle. The freshwater obligate photoautotrophic cyanobacterium Synechococcus elongatus PCC 7942 is a model strain used in cyanobacterial circadian research (Cohen and Golden, 2015). Research based on this cyanobacterium established the proteins KaiA, KaiB and KaiC as the core oscillators of the clock system (Ishiura, 1998; Iwasaki, 1999). KaiC 
performs autokinase, autophosphatase and ATPase functions that are modulated by interaction with KaiA and KaiB. The rhythmic phosphorylation pattern of KaiC functions as a marker of the clock phase and controls rhythmic gene expression. To transfer time information to cellular functions, the oscillator is linked to a two-component regulatory system consisting of the histidine kinase Sas A and the DNA-binding response regulator RpaA (Gutu and O'Shea, 2013). During the day, KaiC is progressively phosphorylated. In its highly phosphorylated state, KaiC activates SasA autophosphorylation activity, resulting in the subsequent phosphorylation of RpaA. In Synechococcus elongatus PCC 7942, phosphorylated RpaA acts as a repressor of dawn-peaking genes and activator of dusk-peaking genes, and the deletion of rpaA leads to the arrest of cells in a dawn-like state (Markson et al., 2013). Although mutations in the KaiABC circadian oscillator do not strongly impair the L/D growth of Synechococcus elongatus PCC 7942, the $\Delta r p a A$ strain is unable to grow in a day/night cycle because it loses viability in D. The described phenotypes of the Synechococcus rpaA mutant strain include imbalances in redox regulation and the accumulation of carbon storage compounds (Diamond et al., 2017; Puszynska and O'Shea, 2017).

Synechocystis sp. PCC 6803 (hereafter Synechocystis) is another frequently used model strain that, in contrast to Synechococcus elongatus PCC 7942, can use glucose for mixotrophic and heterotrophic growth and can tolerate high salinities (Rippka et al., 1979; Kirsch et al., 2019). It contains the KaiA, KaiB1 and KaiC1 proteins with high similarity to the Synechococcus oscillator proteins. Moreover, the Synechocystis genome encodes two additional copies of the kaiC (kaiC2 and kaiC3) and kaiB (kaiB2 and kaiB3) genes (Wiegard et al., 2013). Köbler et al. (2018) showed that the homologous SasARpaA two-component system of Synechocystis interacts only with KaiC1 but not KaiC2 or KaiC3. This specificity of the SasA-RpaA system for KaiC1 suggests that the additional Kai components of Synechocystis may use different output elements ( Figure 1).

True circadian oscillations, which continue after shifting the cells into a constant environment, may not be active in Synechocystis, but robust transcriptional rhythmicity in a diurnal light regime has been described (Beck et al., 2014). Diurnal oscillations depend on daily recurring external stimuli sustaining a $24 \mathrm{~h}$ period. In Synechocystis, the deletion of the kaiABlCl gene cluster and the response regulator gene rpaA, caused defects in cell viability in a 12-hour L/12-hour D cycle, especially under mixotrophic conditions. Furthermore, in complete D, neither mutant strain was able to grow heterotrophically, whereas wild-type growth was slow but observable (Dörrich et al., 2014; Köbler et al., 2018). Surprisingly, DNA microarray analyses of both mutant strains demonstrated a considerable difference in their transcriptome responses to a switch from night to day. The overlap in the transcriptional responses between the mutants was only $50 \%$ in studies performed under identical conditions with a similar microarray design (Dörrich et al., 2014; Köbler et al., 2018). The overlap in the transcriptional changes contained mRNAs encoding regulatory proteins, such as ribonuclease $\mathrm{E}$ (rne), phytochrome 1 (cphl), and the sigma factor SigE (sigE). Furthermore, the $\triangle k a i A B l C l$ and $\triangle$ rpaA mutations affected mRNAs of both gnd encoding the 6-phosphogluconate dehydrogenase and talB encoding the transaldolase. These enzymes are a part of the oxidative pentose phosphate pathway (OPPP), which is activated during the night and converts glucose-6-phosphate (glucose-6P) into pentose- and other sugar phosphates, thereby providing NADPH reduction equivalents (Makowka et al., 2020). In contrast to Gnd, TalB is also active in the Calvin-Benson-Bassham (CBB) cycle during the day. In addition to the common effects of the two mutants, the $\Delta r p a A$ mutant activates in $\mathrm{L}$ the transcription of many genes known to be involved in acclimation to low inorganic carbon $\left(\mathrm{C}_{\mathrm{i}}\right)$ concentrations, i.e., bicarbonate and $\mathrm{CO}_{2}$. In D, in $\triangle r p a A$, but not $\triangle k a i A B 1 C 1$, we observed a decreased transcript accumulation of two enzymes (PhaC/PhaE) involved in NADPH-consuming synthesis (via acetoacetyl-CoA reductase $\mathrm{PhaB}$ ) of the storage compound polyhydroxybutyrate (PHB), which is produced from glycogen under unbalanced nutrient conditions in response to nitrogen starvation (Koch 
et al., 2019, 2020). Werner et al., (2019) performed very detailed metabolite profiling during a sinusoidal diurnal cycle with a light intensity peak in the middle of the day. These authors found highly concerted oscillations of metabolites across the cycle, verifying that Synechocystis performs carbon fixation and protein and nucleotide synthesis during the day. Surprisingly, the authors reported a sharp oscillation in the amount of insoluble carbohydrates at approximately the L-to-D switch. Cyanobacteria store fixed carbon mainly as glycogen, which is then degraded at night using OPPP. Further glycolytic routes, including the Entner-Douderoff (ED) pathway, which branches off the OPPP, and the EmbdenMeyerhof-Parnas (EMP) pathway, have been identified (Chen et al., 2016).

The current study aims to investigate how the differential regulation of gene expression in $\triangle$ kaiABlCl and $\triangle r p a A$ mutants impacts Synechocystis metabolism. We focused on these two mutants because they showed a growth phenotype in a L-D cycle in contrast to a $\triangle \mathrm{kaiC} 3$ strain which had no growth defect under these conditions and a kaiB2C2 mutation which turned out to be lethal (Dörrich et al., 2014). For this purpose, targeted and untargeted metabolic profiling was applied to reveal the changes in primary metabolites in the middle of the $\mathrm{D}$ and L phases. The same time points were investigated in a previous transcriptome analysis of the $\triangle k a i A B l C l$ and $\triangle r p a A$ mutants. The identified differences in the metabolic responses clearly indicate that mainly the lack of the RpaA regulator impacts primary metabolism and the metabolic switch between L and D growth.

\section{Material and Methods}

\subsection{Cyanobacterial strains, culture and sampling conditions}

For standard cultivation, the Synechocystis sp. PCC 6803 wild-type (PCC-M, resequenced, Trautmann et al., 2012) and mutant strains were grown photoautotrophically under constant illumination with $75 \mu \mathrm{mol}$ photons $\mathrm{m}^{-2} \mathrm{~s}^{-1}$ white light at $30^{\circ} \mathrm{C}$ in BG11 medium (Rippka et al., 1979) supplemented with $10 \mathrm{mM}$ TES buffer ( $\mathrm{pH} 8$ ) in flasks. For the L/D experiments, the precultures were grown in constant light and diluted to $\mathrm{OD}_{750 \mathrm{~nm}}$ 0.4-0.6 before transfer to alternating 12-hour L/12-hour D cycles with $75 \mu \mathrm{mol}$ photons $\mathrm{m}^{-2} \mathrm{~s}^{-1}$ during the $\mathrm{L}$ phase. Depending on the experimental setup, air was supplemented with $\mathrm{CO}_{2}(1 \%, \mathrm{v} / \mathrm{v})$, and illumination was increased to $150 \mu \mathrm{mol}$ photons $\mathrm{m}^{-2} \mathrm{~s}^{-1}$ after the liquid cultures reached an $\mathrm{OD}_{750 \mathrm{~nm}}$ of $\sim 1$. The wild-type and mutant strains were cultured in triplicate. The samples were collected in the middle of the first D phase and the following L phase $5.5 \mathrm{~h}$ after each transition in the exponential growth phase if not described otherwise.

\subsection{RNA isolation and Northern blot hybridization}

The RNA isolation was performed following Pinto et al., (2009). For sampling, $20 \mathrm{ml}$ of culture were filtered through a Supor- 800 membrane filter $(0.8 \mathrm{~mm}$, Pall), vortexed in $1.6 \mathrm{ml}$ PGTX for $30 \mathrm{~s}$ and immediately frozen in liquid nitrogen. The samples were stored at $-80^{\circ} \mathrm{C}$. After thawing the samples on ice, the samples were heated at $95^{\circ} \mathrm{C}$ for $5 \mathrm{~min}$. The lysis was supported by vortexing during the incubation. After cooling the samples on ice for $5 \mathrm{~min}$, the samples were incubated at room temperature (RT) for $10 \mathrm{~min}$. Then, $200 \mu \mathrm{l}$ of cold BCP were added, vortexed for $30 \mathrm{~s}$ and stored at RT for $15 \mathrm{~min}$. Next, the phases were separated by centrifugation at $3100 \times \mathrm{g}$ for $15 \mathrm{~min}$ at $4^{\circ} \mathrm{C}$. The aqueous phase was transferred into a new reaction tube, and the reaction was repeated once more with 1 volume of cold BCP. The samples were centrifuged at $21130 \times \mathrm{g}$ for $15 \mathrm{~min}$ at $4{ }^{\circ} \mathrm{C}$, followed by precipitation with 1 volume of isopropanol and storage of the sample overnight at $-20^{\circ} \mathrm{C}$. The samples were centrifuged at $21130 \times \mathrm{g}$ for $1 \mathrm{~h}$ at $4^{\circ} \mathrm{C}$, the resulting pellet was washed with $75 \% \mathrm{EtOH}$, and centrifugation was

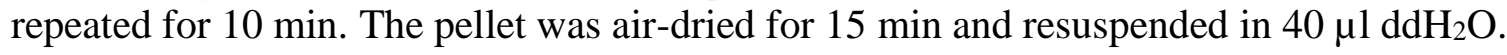


To separate the RNA, $10 \mu \mathrm{g}$ were used for electrophoresis on a $1.5 \%$ denaturing agarose-formaldehyde gel. The gel was blotted onto a Roti-Nylon plus membrane (Roth). The DNA fragment used for probe generation was amplified from wild-type DNA using the oligonucleotides sbtA-fw (5, CAATCCCCATAAATTTCAACCAAGGAGAC 3') and sbtA-rev (5' TAATACGACTCACTATAGGGAGAGCCAAGGGCGGCAATAACCATC 3'). The probe was produced with a Rediprime II labeling kit (GE Healthcare). Hybridization was performed as described by Dienst et al. (2008), and the signals were detected using a Typhoon FLA 9500 (GE Healthcare).

\subsection{Immunoblot analysis of RubisCO}

The wild-type and mutant cultures of Synechocystis were diluted to OD $750 \mathrm{~nm} 0.6$ and cultivated in an L/D cycle. $5 \mathrm{ml}$ of WT and mutant cultures of Synechocystis were harvested by centrifugation at $4000 \times \mathrm{g}$ for $2 \mathrm{~min}$ at $4^{\circ} \mathrm{C}$. After the pellet was resuspended in PBS buffer $\left(8.6 \mathrm{mM} \mathrm{Na} 2 \mathrm{PO}_{4}, 1.8 \mathrm{mM}\right.$ $\mathrm{KH}_{2} \mathrm{PO}_{4}, 137 \mathrm{mM} \mathrm{NaCl}, 2.7 \mathrm{mM} \mathrm{KCl}, \mathrm{pH}$ 7.5), the cells were disrupted in a cell mill using glass beads. The crude extract was obtained by centrifugation at $500 \times \mathrm{g}$ for $5 \mathrm{~min}$ at $4{ }^{\circ} \mathrm{C}$, and different protein amounts of the crude extract were subjected to SDS-PAGE. The immunodetection was performed using anti-RbcL (Agrisera, Sweden) and secondary anti-rabbit (Thermo Fisher Scientific Inc., USA) antibodies.

\subsection{Quantification of the PHB content}

The intracellular PHB content was measured as described by Taroncher-Oldenburg et al. (2000). The wild-type and mutant cultures were adjusted to OD750 $\mathrm{nm} 0.2$ and then grown in L/D cycles supplemented with $\mathrm{CO}_{2}$ as described above. The samples were collected in the $7^{\text {th }} \mathrm{L} / \mathrm{D}$ cycle $1 \mathrm{~h}$ before the $\mathrm{L}$ or $\mathrm{D}$ transitions. Approximately $15-35 \mathrm{ml}$ of cell culture were harvested by centrifugation $\left(4000 \times \mathrm{g}, 10 \mathrm{~min}, 4^{\circ} \mathrm{C}\right.$ ), washed once with $\mathrm{H}_{2} \mathrm{O}$ and dried overnight at $85^{\circ} \mathrm{C}$. To break the cells and convert PHB, the pellet was boiled in $1 \mathrm{ml}$ concentrated $\mathrm{H}_{2} \mathrm{SO}_{4}$ for $1 \mathrm{~h}$. Then, $100 \mu \mathrm{l}$ were transferred to $900 \mu \mathrm{l}$ of $0.014 \mathrm{M} \mathrm{H}_{2} \mathrm{SO}_{4}$. To pellet the cell debris, the sample was centrifuged at $17000 \times \mathrm{g}$ for $5 \mathrm{~min}$ before $500 \mu \mathrm{l}$ of the supernatant were diluted in $500 \mu \mathrm{l}$ of $0.014 \mathrm{M} \mathrm{H}_{2} \mathrm{SO}_{4}$. The centrifugation was repeated, and the supernatant was analyzed via Acquity UPLC BEH (WATERS) with a reversedphase C18 column (Eurosphere II, 100 by $2.1 \mathrm{~mm}$, Knauer). The separation was performed using increasing concentrations of acetonitrile in $0.1 \%$ formic acid. Commercially available crotonic acid was used as a standard in parallel with a conversion ratio of 0.893 (Koch et al., 2019).

\subsection{Nontargeted metabolome profiling analysis by gas chromatography-mass spectrometry (GC-MS)}

The metabolome profiling of the metabolite fraction enriched with primary metabolites was performed as previously described (Kopka et al., 2017). $20 \mathrm{ml}$ of the cultures were sampled by fast filtration onto $47 \mathrm{~mm}$ diameter glass microfiber filters of a $1.2 \mu \mathrm{m}$ pore size (GE Healthcare, Little Chalfont, England), followed by immediate shock freezing. The complete process was less than 30 s. Samples equivalent to approximately $20 \mathrm{ml}$ of $\mathrm{OD}_{750 \mathrm{~nm}}=0.7-1.0$ cultures were extracted for $15 \mathrm{~min}$ at $37^{\circ} \mathrm{C}$ by $1 \mathrm{ml}$ methanol:chloroform:diethylamine 2.5:1.0:1.0 (v/v/v). The extraction mixture contained 0.02 $\mathrm{mgml}^{-1}{ }^{13} \mathrm{C}_{6}$-sorbitol, which was used as an internal standard (Erban et al., 2020). A polar liquid phase was obtained by adding $400 \mu \mathrm{l}$ distilled water and centrifuging for $5 \mathrm{~min}$ at 14,000 rpm in an Eppendorf 5417 microcentrifuge. The top liquid phase, $\sim 700 \mu 1$, was dried by vacuum centrifugation overnight in 1.5-mL microcentrifugation tubes and stored at $-20^{\circ} \mathrm{C}$.

The chemical derivatization for the GC analysis, GC-MS-based metabolite profiling and retention index standardization were performed as described by Erban and coauthors (2020) using electron 
impact ionization time-of-flight mass spectrometry (GC/EI-TOF-MS). The GC/EI-TOF-MS system was an Agilent 6890N24 (Agilent Technologies, Waldbronn, Germany) gas chromatograph hyphenated to a LECO Pegasus III time-of-flight mass spectrometer (LECO Instrumente GmbH, Mönchengladbach, Germany). The chromatograms were acquired, and the baseline was corrected by ChromaTOF software (LECO Instrumente GmbH, Mönchengladbach, Germany). The metabolites were annotated by manual supervision using TagFinder software (Luedemann et al., 2008), NIST2017 version $2.3^{1}$ and the mass spectral and retention time index (RI) reference data of the Golm Metabolome Database (Kopka et al., 2005). GC/EI-TOF-MS was normalized to the internal standard ${ }^{13} \mathrm{C}_{6}$-sorbitol, $\mathrm{OD}_{750 \mathrm{~nm}}$ of the culture and volume of the sample.

\subsection{Targeted metabolome analysis by liquid chromatography-mass spectrometry (LC-MS)}

The sampling of the cells was performed precisely as described in the previous paragraph. Low molecular mass compounds were extracted from the cells with $2 \mathrm{ml}$ of ethanol (80\%, HPLC grade, Roth, Germany) at $65^{\circ} \mathrm{C}$ for $2 \mathrm{~h}$. One microgram of carnitine was added to each sample as an internal standard to correct for losses during the extraction and sample preparation. After centrifugation, the supernatants were collected and freeze-dried. The dry extracts were dissolved in $1 \mathrm{ml} \mathrm{MS}$-grade water and filtered through $0.2 \mu \mathrm{m}$ filters (Omnifix®-F, Braun, Germany). The cleared supernatants were analyzed using a high-performance liquid chromatograph mass spectrometer system (LCMS-8050, Shimadzu, Japan). In brief, $1 \mu 1$ of each extract was separated on a pentafluorophenylpropyl (PFPP) column (Supelco Discovery HS FS, $3 \mu \mathrm{m}, 150 \times 2.1 \mathrm{~mm}$ ) with a mobile phase containing $0.1 \%$ formic acid. The compounds were eluted at a rate of $0.25 \mathrm{ml} \mathrm{min}-1$ using the following gradient: $1 \mathrm{~min} 0.1 \%$ formic acid, $95 \%$ distilled water, $5 \%$ acetonitrile, within 15 min linear gradient to $0.1 \%$ formic acid, $5 \%$ distilled water, $95 \%$ acetonitrile, $10 \mathrm{~min} 0.1 \%$ formic acid, $5 \%$ distilled water, and $95 \%$ acetonitrile. Aliquots were continuously injected into the MS/MS part and ionized via electrospray ionization (ESI). The compounds were identified and quantified using the multiple reaction monitoring (MRM) values given in the LC-MS/MS method package and the LabSolutions software package (Shimadzu, Japan). Defined standard mixes of amino acids and organic acids (usually $1 \mathrm{ng}$ of the specific metabolite was injected) were analyzed in the same run to calculate the absolute contents per sample. Then, the metabolite levels were normalized to the detected amount of the internal standard carnitine, sample volume and $\mathrm{OD}_{750 \mathrm{~nm}}$.

\subsection{NAD(P) and NAD(P)H analyses}

$\mathrm{NAD}(\mathrm{P})$ and $\mathrm{NAD}(\mathrm{P}) \mathrm{H}$ were extracted from approximately $8 \mathrm{ml}$ of $\mathrm{OD}_{750 \mathrm{~nm}}=0.7-1.0$ cultures sampled onto $47 \mathrm{~mm}$ diameter glass microfiber filters of a $1.2 \mu \mathrm{m}$ pore size (GE Healthcare, Little Chalfont, England), followed by immediate shock freezing. The quantification of NAD, NADP and NADPH was performed as previously described using enzymatic cycling assays (Zhang et al., 2020). The samples used for the NAD and NADP analyses were extracted by $500 \mu \mathrm{L}$ extraction solution containing $0.1 \mathrm{M}$ $\mathrm{HClO}_{4}$ in $50 \%$ ethanol; the NADPH extraction was performed by $0.1 \mathrm{M} \mathrm{KOH}$ in $50 \%$ ethanol (Tamoi et al., 2005). The samples were thoroughly vortexed with intermittent sonication in an ice bath 3 times for $45 \mathrm{sec}$ each, followed by a final incubation on ice for $10 \mathrm{~min}$. After centrifugation for $10 \mathrm{~min}$ at $14,000 \mathrm{rpm}$ at $4^{\circ} \mathrm{C}, 100 \mu \mathrm{L}$ of extract were transferred to a microcentrifuge vial and heat inactivated at $95^{\circ} \mathrm{C}$ for $2 \mathrm{~min}$. Then, the samples were cooled on ice and neutralized either by $100 \mu \mathrm{L}$ of $0.1 \mathrm{M} \mathrm{KOH}$ in $0.2 \mathrm{M}$ Tris $\mathrm{HCl} \mathrm{pH} 8.4$ for the NAD and NADP quantitation or $100 \mu \mathrm{L}$ of $0.1 \mathrm{HClO}_{4}$ in $0.2 \mathrm{M}$ Tris $\mathrm{HCl} \mathrm{pH} 8.4$ for the NADPH analyses. The quantitation was performed using 25 or $40 \mu \mathrm{L}$ volumes of the neutralized extracts used for the previously described cycling assays (Zhang et al., 2020). Care was

\footnotetext{
${ }^{1}$ http://chemdata.nist.gov/ 
taken to adjust the samples and quantitative calibration standards to identical final ethanol concentrations (Supplemental Table S3). In this study, the quantities of NAD, NADP and NADPH in the biological samples were sufficient for the quantitation. NADH quantitation was attempted but failed due to low concentrations in the biological samples. The analyses were biologically replicated using 3 independent cultures. The cultures were probed by 1 (NADPH) or 2 (NAD and NADP) analytical repeats, i.e., parallel samplings from the same culture. The quantitative data of the analytical repeats were averaged prior to the statistical analysis of the 3 independent biological replications.

\subsection{Metabolomic data analysis}

All metabolomics data analyses, the hierarchical cluster analysis (HCA) by Pearson's correlation and average linkage, the principal component analysis (PCA), the two factorial analyses of variance (ANOVA), the statistical testing, e.g., Tukey tests, and the data visualization, were performed using RStudio statistical programming ${ }^{2}$ and the program packages "impute", "multcompView", "ggplot2", "eulerr", and "https://github.com/MSeidelFed/RandodiStats_package.git". The bootstrapping analysis of the HCA tree was performed using the "pvclust" R package, and the node confidence was evaluated by "approximately unbiased" and "ordinary" bootstrap probabilities.

The relative quantification data from the GC-MS profiling analyses and quantitative data from the LCMS analyses were maximum scaled per metabolite prior to further analyses. For the global data analyses, the missing values in the data set were replaced by the k-nearest neighbors algorithm (k-NN) and autoscaled. For the statistical data analyses, the data matrices were $\log _{10}$-transformed after the missing data replacement by a small value, i.e., $10^{-9}$. The statistical analyses were performed using the log-transformed data. The relative concentration changes are reported as fold changes (FCs). The FCs of the metabolites that were detectable under one condition and undetectable under the compared condition, e.g., FCs comparing L to D responses or FCs comparing mutant to wild type (Supplemental Table S1-S2), are reported. The significance analyses were replaced by presence and absence calls, and the FCs were scored as either significant increases or decreases.

For the analyses comparing the data to previous studies, the data were merged according to the technology platform, namely, GC-MS profiling and LC-MS quantification. Merging across technology platforms was only performed if data from the same technology platform were not available. The replicate data were averaged per study maximum-scaled, and the missing values were replaced by the k-NN method. The final data matrices were log transformed and autoscaled prior to the subsequent PCA.

\section{$3 \quad$ Results}

\section{1 $\mathrm{CO}_{2}$ availability can compensate for the growth defects of $\triangle k a i A B 1 C 1$ and $\triangle r p a A$ mutants in $\mathbf{L} / \mathbf{D}$ cycles}

Previous analyses revealed the essential role of the clock homologs KaiAB1C1 and RpaA in the viability of Synechocystis in L/D cycles and in the D under heterotrophic conditions (Dörrich et al., 2014; Köbler et al., 2018). Although the phenotypes of the corresponding mutants were very similar, microarray analyses revealed substantial differences in gene expression between these strains, such as an accelerated $\mathrm{C}_{\mathrm{i}}$ response that was detected only in the $\triangle r p a A$ mutant (Köbler et al., 2018). To determine whether $\mathrm{C}_{\mathrm{i}}$-responsive genes are still upregulated in $\triangle r p a A$ even under high $\mathrm{CO}_{2}$ conditions,

\footnotetext{
${ }^{2}$ https://rstudio.com/
} 
we analyzed the expression of $s b t A$, which encodes a sodium-dependent bicarbonate transporter. Using Northern blot analysis, we did not detect a difference in the $s b t A$ mRNA levels between the wild-type and mutant strains under elevated $\mathrm{CO}_{2}$ concentrations, suggesting that $\mathrm{C}_{\mathrm{i}}$-responsive genes are not a direct target of the RpaA transcription factor (Supplementary Figure S1). Furthermore, we examined the response of the mutant strains to $\mathrm{CO}_{2}$ availability by growing cells under low (ambient) and high $\mathrm{CO}_{2}(1 \%)$ conditions in liquid cultures (Figure 2). Similar to previous studies in which viability assays were performed using agar plates, the $\triangle$ kaiABlCl and $\triangle r p a A$ strains showed a pronounced growth defect in L/D cycles (Figure 2A). The curves also revealed that growth arrest mainly appeared when the mutant cultures left the exponential growth phase (Figure 2A). As an organic carbon source, glucose had an additional inhibitory effect on the viability of the mutant cells (Dörrich et al., 2014; Köbler et al., 2018), whereas an increase in $\mathrm{CO}_{2}$ availability could largely align the growth of the mutant cultures with the wild-type cultures in an L/D cycle (Figure 2B). Our previous viability analyses under mixotrophic/heterotrophic conditions and the growth assays under elevated $\mathrm{CO}_{2}$ availability in this study indicate that defects in the Synechocystis Kai system led to an imbalance in carbon acquisition and changes in central carbon metabolism.

\subsection{PHB accumulation is reduced in Synechocystis $6803 \Delta$ kaiAB1C1 and $\triangle r p a A$ mutants}

In addition to the altered $\mathrm{C}_{\mathrm{i}}$ acclimation response, the expression of two genes encoding polyhydroxybutyrate (PHB) synthase subunits ( $p h a C$ and phaE) was highly decreased in the $\Delta r p a A$ strain in D (Köbler et al., 2018). A similar tendency in phaCE transcript accumulation was also detected in a microarray analysis of the $\triangle$ kaiABlCl strain; this tendency was less pronounced and did not match our significance criteria (Dörrich et al., 2014). In the previous microarray analyses, the cells were collected during the exponential growth phase and were grown under low $\mathrm{CO}_{2}$ conditions, where $\mathrm{PHB}$ synthesis is very low, and the amount of this storage compound was not measurable. Therefore, we evaluated the impact of KaiAB1C1 and RpaA on PHB production under high $\mathrm{CO}_{2}$ conditions during the stationary growth phase. Under these conditions, the $\triangle k a i A B 1 C 1$ and $\triangle r p a A$ strains did not show growth arrest in an L/D cycle and exhibited only a slightly lower growth rate than the wild type (Figure 2B). Consistent with the lowered pha gene expression, both strains showed a strong reduction in PHB accumulation in the $\mathrm{D}$ and $\mathrm{L}$ phases, suggesting that the high $\mathrm{CO}_{2}$ availability was unable to compensate for the defect in PHB production (Figure 3). PHB synthesis depends on many factors, such as the C:N ratio and nutrient starvation. A complex relationship links PHB synthesis and utilization to central metabolic pathways (Ciebiada et al., 2020; Koch et al., 2020). Therefore, the transcriptomic changes might not fully represent the role of KaiAB1C1 and RpaA in cellular nightand daytime functions, which led us to analyze the metabolic changes in the two corresponding mutants.

\subsection{Global metabolic analysis of the $\triangle r p a A$ and $\triangle k a i A B 1 C 1$ mutants after L/D entrainment}

The previous investigations and data presented above suggest that the KaiAB1C1 proteins along with the RpaA regulator impact primary metabolism in Synechocystis and growth specifically under low $\mathrm{C}_{\mathrm{i}}$ conditions in an L/D cycle (Figure 2A). Therefore, the metabolome of the two mutants was analyzed after an L-to-D transition under ambient $\mathrm{CO}_{2}$ supply. Independent, exponentially grown precultures in ambient air were adjusted to similar optical densities of approximately 0.6 and sampled either in the middle of the first $\mathrm{D}$ phase at $5.5 \mathrm{~h}$ of the diurnal cycle or in the middle of the following $\mathrm{L}$ phase at $17.5 \mathrm{~h}$ (Figure 4A). The mutants and wild type exhibited similar growth rates during this precultivation under continuous L (Supplementary Figure S2). The time points were selected to avoid the expected rapid changes in metabolism directly after illumination shifts (Werner et al., 2019), to minimize the secondary effects of the growth arrest of the mutants in L/D cycles and to correlate the metabolic data 
of this study to previously reported transcriptomic data (Dörrich et al., 2014; Köbler et al., 2018) and to metabolic data published for Synechococcus elongatus PCC 7942 (Diamond et al., 2017).

We analyzed 80 metabolites from central metabolism by relative quantification of a metabolite fraction enriched for primary metabolites via gas chromatography-mass spectrometry (GC-MS)-based profiling or absolute quantification via liquid chromatography-mass spectrometry (LC-MS) (Supplemental Table S1). In addition to the identified metabolite information, the nontargeted metabolite profiling yielded a set of yet unidentified metabolites and mass features that contributed to the global analyses of the metabolic phenotypes in this study. The hierarchical cluster and principal component analyses (PCA) indicated that the switch between $\mathrm{D}$ and $\mathrm{L}$ was the major experimental factor affecting the metabolite phenotypes (Figure 4B) with a contribution of $31.3 \%$ to the total variance according to PC1 (Figure 4C, Supplemental Figure S3). The wild type and two mutants clustered with high bootstrapping confidence of their independent replicate profiles (Figure 4B). The metabolic profiles of the $\triangle$ kaiABlCl and $\triangle r p a A$ mutants differed from those of the wild type in the D and L phases (Figure 4B-C). However, the metabolic phenotype of the $\triangle r p a A$ mutant differed more, especially in $\mathrm{D}$, when the deviations were stronger and amounted to a distinct PC3. PC3 differentiated $\triangle r p a A \mathrm{D}$ metabolism from all other sample profiles by $11.2 \%$ of the total variance (Figure 4C). The D metabolism of $\triangle r p a A$ appeared to be between that of the wild type D and L states (Figure 4C) with a tendency towards wild type in L (Figure 4B).

Molecular studies have suggested that the $\Delta r p a A$ mutant of Synechococcus elongatus PCC 7942 may be arrested in a dawn-like expression state (Markson et al., 2013). In wild-type Synechocystis cells, a set of metabolites accumulates rapidly following the onset of illumination after dawn and declines in the course of the L phase, which may indicate a morning state of metabolism (Werner et al., 2019). These "morning metabolites" include malate, fumarate, amino acids, serine, valine, isoleucine, glutamine, aspartate, arginine, and nucleotide-related metabolites, such as cytidine, cytidine monophosphate (CMP), cytidine triphosphate (CTP), uridine monophosphate (UMP), guanosine monophosphate (GMP), guanosine triphosphate (GTP), hypoxanthine, inosine, and inosine monophosphate (IMP) (Werner et al., 2019). Although our study investigated the metabolome in the middle of the day, two "morning metabolites", namely, aspartate and serine, significantly accumulated (Tukey test, $P<0.05$ ) in the illuminated $\triangle r p a A$ mutant of Synechocystis, whereas many more metabolites shifted to higher values in the wild-type cells. In contrast, isoleucine and valine significantly accumulated during D in $\triangle r p a A$ (Figure 5). For a global comparison of our metabolite profiles and a previous diurnal characterization of Synechocystis central metabolism (Werner et al., 2019), we aligned the 47 commonly monitored metabolites. The PCA of the combined and separately scaled data sets revealed PC1, which had a high percentage of shared total variance, i.e., 45.8\%. PC1 separated the wild-type L metabolomes from the D metabolomes of the two studies in a nonlinear manner (Supplemental Figure S4). Colinearity indicated high agreement in the L/D-induced metabolome changes between the two studies. However, PC2, which accounted for $16.8 \%$ of the total variance, rendered a more precise alignment, and a comparison of the sampled time points between the two studies is impossible. Further comparative meta-analysis was abandoned because the studies clearly used different growth conditions. For example, high peaking light intensity at $1600 \mu \mathrm{mol}$ photons $\mathrm{m}^{-2} \mathrm{~s}^{-1}$ and high $5 \% \mathrm{CO}_{2}$ were applied in a previous study (Werner et al., 2019), whereas low light of $50 \mu \mathrm{mol}$ photons $\mathrm{m}^{-2} \mathrm{~s}^{-1}$ and ambient $\mathrm{CO}_{2}$ were applied in this study.

A global correlation analysis of the metabolites was performed using all mutant and wild-type data in the current study. We present this analysis as a Pearson's correlation matrix that analyzes the relative concentration changes in all monitored metabolites (Supplemental Figure S5). This analysis indicates a central group of highly correlated metabolites that contained metabolites of lower glycolysis, 3- 
phosphoglycerate (3PGA), 2-phosphoglycerate (2PGA), phosphoenolpyruvate (PEP), and of photorespiration, 2-phosphoglycolate (2PG), serine and glycerate. Two additional large groups contained metabolites that were inversely correlated between the groups but partially formed highly correlated subsets within each group. The first group comprised most amino acids and the oxidative (C6-C5) branch of tricarboxylic acid (TCA) metabolism. The second group contained predominantly metabolites of nucleic acid metabolism and the reductive (C4) branch of TCA metabolism (Supplemental Figure S5).

\section{4 $\triangle k a i A B 1 C 1$ and $\triangle r p a A$ mutations attenuate metabolic $\mathrm{L} / \mathrm{D}$ responses}

Our study revealed that 46 metabolites, i.e., more than half of all monitored compounds, significantly (Tukey test, $P<0.05$ ) differed between the mutants and wild type. A large overlap of 21 metabolic changes during the L/D cycle was observed between the two mutants (Figure 6). This finding supported the hypothesis that the KaiAB1C1 system contributes to the diurnal control of central metabolism mostly via RpaA-mediated output signaling (Figure 1). Most of the common metabolic defects were apparent in both phases (12), except for one defect, which was limited to the L phase (9). Deviations specific to only one mutant suggested a small RpaA-independent metabolic signaling component in the KaiAB1C1 system, with three metabolic changes in D, one metabolic change in $\mathrm{L}$ and one metabolic change in both. In contrast, the KaiAB1C1-independent changes in the $\Delta r p a A$ mutant (20) exhibited a preference for RpaA-specific metabolic defects in D (10) (Figure 6), which is consistent with the closer clustering of $\triangle r p a A \mathrm{D}$ with the L samples of all strains (see Figure 4). The larger impact of the RpaA deficiency may be explained by its functionality as a transcription factor with partly retained functions in the absence of Kai-based control and the KaiAB1C1-independent additional functions of RpaA that are established via protein-protein interactions (Köbler et al., 2018). For example, RpaA interacts with $\mathrm{RpaB}$, which is the transcriptional regulator responding to environmental changes, including L/D transitions, via concomitant redox regulation (Riediger et al., 2019) (Figure 1).

The set of 46 mutant-dependent metabolites included unidentified compounds and metabolites with small but significant pool size changes (Supplemental Table S1). To focus our data analysis, for the following analysis, we selected only identified metabolites that were significantly (Tukey test, $P \leq$ 0.05) responsive to the L/D change (Figure 5). From this subset, we distinguished five groups of L/Dresponsive metabolites (L/D groups) according to their relative changes in the wild type compared to the mutant defects. L/D group I contained 17 metabolites (mostly amino acids) that accumulated in the wild type in L compared to D (Figure 5). A second set of 11 metabolites, i.e., L/D group II, exhibited inverse changes in the wild type compared to group I and contained metabolites, e.g., organic acids, that decreased in L compared to D. Only a small set of metabolites from these two groups, namely, arginine, aspartate, proline (L/D group I) and malate (L/D group II), maintained L/D responsiveness in the mutants. KaiAB1C1 and especially the RpaA deficiency attenuated the L/D responsiveness of a large fraction of L/D-responsive metabolites in both groups. Many attenuated changes were shared between the $\triangle r p a A$ and $\triangle k a i A B 1 C l$ mutants. However, the $\triangle r p a A$ mutant clearly had more metabolites with attenuated L/D responsiveness (Figure 5).

Furthermore, we discovered additional but rarer metabolite response patterns supporting the hypothesis that central metabolic responses to a switch between photoautotrophy and dark-induced heterotrophy are all but passive. These metabolic responses are enhanced and modulated by KaiAB1C1 and/or RpaA. Glutamate (L/D group III) represented one of these rarer patterns as it accumulated in the wild type in the L phase and had an inverted L/D response only in the $\Delta r p a A$ mutant. Similarly, pyruvate and histidine (L/D group IV) were depleted in the wild type in the L and accumulated in the $\Delta r p a A$ 
mutant (Figure 5). Some metabolites (L/D-group V), e.g., N-acetyl-glutamate, glutamine, ornithine1,5-lactam, lysine, citrate/isocitrate, TMP and CMP, even gained significant L/D responsiveness in one or both mutants (Figure 5). Further central metabolites, e.g., 2PGA and PEP, exhibited response patterns similar to those of L/D group IV but had only small effect sizes and failed to pass the significance threshold (Supplemental Table S1).

\section{5 $\triangle k a i A B 1 C 1$ and $\triangle r p a A$ mutations affect central metabolic pathways}

The preceding part of our metabolic analysis highlighted only one factor of our two-factorial study, namely, the change in illumination and the respective photoautotrophy to heterotrophy transitions of metabolism. However, the attenuation of the L/D response ratios compared to those of the wild type can have multiple alternative causes. Among other causes, the loss of L/D responsiveness can be explained by a deficiency to accumulate a metabolite pool that should increase upon illumination or a failure to decrease metabolites upon $\mathrm{D}$ transition that are high in L. The latter explanation applied in the analysis of the $\triangle r p a A$ mutant to a large set of metabolites from L/D-group I (Figure 5, relativeabundance section). Specifically, a set of amino acids, including alanine and the branched chain amino acids valine, isoleucine, or leucine, retained high concentrations upon the D transition (Figure 5). Similarly, 2PG lost L/D responsiveness in both the $\triangle r p a A$ and the $\triangle$ kaiABlC1 mutants, but $\triangle$ kaiABlC1 showed a nonsignificant illumination-independent increase compared to the extreme and highly significant accumulation of $2 \mathrm{PG}$ in the $\triangle r p a A$ mutant (Figure 7). In the following section, we specifically compared the mutant defects with the wild-type defects (Supplemental Table S2), thereby focusing on pathways that contain metabolites with large concentration changes to identify the fundamental metabolic aspects associated with the mutant defects.

Lower glycolysis. The two products of RubisCO, namely, the carboxylation product 3PGA and the oxygenation product $2 \mathrm{PG}$, were highly elevated in the $\mathrm{L}$ samples of the $\Delta r p a A$ mutant and, to a lesser extent, in the $\triangle \mathrm{kaiABlCl}$ mutant (Figure 7). These increases suggested enhanced RubisCO activity. To support this hypothesis, we analyzed the amount of RubisCO in the mutants by a Western blot analysis (Figure 8). Compared to the wild type, the $\triangle k a i A B 1 C 1$ and $\triangle r p a A$ cells accumulated more of the RbcL subunit in L and D, suggesting that the higher amounts of 3PGA and 2PG could indeed be based on a cellular increase in the RubisCO concentration. The higher amounts of 3PGA in the $\triangle r p a A$ mutant were consistently associated with lower glycolysis with accumulating 2PGA and PEP concentrations in this strain. These findings suggest an activation of carbon utilization from the CBB cycle in the direction of lower glycolysis and towards the oxidative branch of the TCA cycle in the absence of RpaA.

The assumption of the preferred use of 3PGA in the illuminated $\triangle r p a A$ mutant by lower glycolysis and not by gluconeogenesis was supported by the day levels of soluble carbohydrates, such as sucrose, which were not significantly changed in the $\Delta r p a A$ cells (Supplemental Table S1). Similar changes in lower glycolysis and carbohydrate metabolism occur in Synechocystis cells shifted from high- to low- $\mathrm{CO}_{2}$-supply (Orf et al., 2015). The imbalance of carbon use in favor of lower glycolysis may be controlled via the newly discovered PII-regulated switch of phosphoglycerate mutase (PGAM) activity via PirC (Orthwein et al., 2021). Previous gene expression studies implied that PirC (S110944) is downregulated in the $\triangle r p a A$ mutant under L conditions but not in $\mathrm{D}$, although these data do not meet our significance criteria (Köbler et al., 2018). Hence, the lower PGAM inhibition in the $\triangle r p a A$ mutant likely stimulates the higher flux to $2 \mathrm{PGA}, \mathrm{PEP}$, and pyruvate compared to the wild type.

The disbalanced carbon utilization in the $\Delta r p a A$ cells extended to the pyruvate family of amino acids, i.e., alanine, and the branched chain amino acids leucine, isoleucine and valine. Hence, pyruvate is 
crucial for metabolism and cannot be depleted without affecting cyanobacteria growth (Kopka et al., 2017). According to its high demand during growth in the L, the pyruvate levels declined in the wild type upon transition from D to L. The pyruvate decrease was consistent with associated increases in the pyruvate amino acid family in the L (Supplemental Table S1). These amino acid responses remained unaltered in the $\triangle k a i A B l C 1$ cells, but alanine, leucine, isoleucine and valine consistently overaccumulated in the darkened $\triangle r p a A$ cells (Figure 9). The deregulation of amino acid biosynthesis in the $\triangle r p a A$ mutant can contribute to the observed pyruvate depletion and may ultimately affect growth.

Photorespiration. The previously mentioned increased amounts of photorespiratory 2PG extended within the pathway towards higher amounts of glycolate and serine but not glycine in the illuminated $\triangle r p a A$ cells (Figure 7). Glycine lost L/D responsiveness in the $\Delta r p a A$ cells, and the balance of the NADH-producing glycine decarboxylase complex (GDC) and serine hydroxymethyltransferase (SHMT) reaction favored serine. Photorespiratory metabolism was not significantly changed in the $\triangle$ kaiAB1C1 mutant, which is consistent with the only marginal increase in 2PG in this mutant. The increased 2PG and glycolate levels indicate an increased flux into the Synechocystis photorespiratory pathway (Eisenhut et al., 2008; Huege et al., 2011) that detoxifies the critical substrate 2PG. The growth defect of the $\triangle r p a A$ cells in the L/D cycle is clearly associated with constitutively elevated $2 \mathrm{PG}$ levels. This observation is consistent with the finding that $2 \mathrm{PG}$ inhibits several key enzymes in plant primary carbon metabolism (e.g., Flügel et al., 2017). Furthermore, Synechocystis uses 2PG accumulation as a metabolic signal that activates the expression of cyanobacterial carbon-concentrating metabolism (CCM) because 2PG stimulates the activator $\mathrm{CmpR}$ and inactivates the repressor protein NdhR (Nishimura et al., 2008; Jiang et al., 2018). CCM components, such as sbtB, ndhD3, ndhF3, сирA, стрA, and стрB (Shibata et al., 2001, 2002; Xu et al., 2008; Orf et al., 2015), are consistently expressed at higher mRNA levels during the L phase in $\triangle r p a A$ cells (Köbler et al., 2018).

Consistent with the previously reported deregulation of photosystem I and phycobilisome gene expression in the D phase (Köbler et al., 2018), the 2PG levels largely remained at increased levels in the nonilluminated $\triangle r p a A$ mutant cells. In contrast, the amount of 3PGA consistently decreased in the $\mathrm{D}$ phase because inorganic carbon assimilation halted. RubisCO is assumed to be inactive during the night, but other sources of 2PG synthesis seem unlikely. An explanation of mistimed 2PG accumulation is proposed by the hypothesis that RubisCO may not be fully inactivated in $\triangle r p a A$ mutant cells during the night, especially because there seems to be substantial overaccumulation of this enzyme in these cells (Figure 8). RubisCO which is not completely inactivated may then preferentially perform oxygenation during the night due to the low $\mathrm{C}_{\mathrm{i}} /$ high $\mathrm{O}_{2}$ intracellular environment. This explanation not only applies to the mutant cells but also seems to apply to the wild type, which fully detoxifies $2 \mathrm{PG}$ in $\mathrm{D}$ but still contains significantly higher amounts of glycolate and glycine at night compared to the day (Figure 7). An alternative explanation of elevated 2PG in D may be a potential carry-over of the high concentration from the preceding L phase. Such an assumption could imply D inhibition of the $2 \mathrm{PG}$ dephosphorylation step in the $\triangle r p a A$ mutant. However, regulation of the $2 \mathrm{PG}$ phosphatase step seems unlikely because of the presence of up to four partially highly promiscuous 2-PG phosphatases within the Synechocystis genome (Rai et al., 2018).

TCA pathways. Our metabolome analysis covered most metabolites of the noncanonical TCA cycle (Figure 10) and associated amino acid biosynthesis pathways that receive carbon building blocks from this cycle (Figure 11). Among cyanobacteria, the TCA cycle is not closed because the 2-oxoglutarate (2OG) dehydrogenase complex is missing. The open nature of the TCA pathways is clearly reflected in our current study by the inverted L/D responses of the TCA cycle metabolites from its reductive 
(C4) and oxidative (C6 and C5) branches. Malate, fumarate and succinate of the reductive branch decreased in $\mathrm{L}$ with large decreases in the malate and fumarate pools in all strains but only minor changes in succinate. This consistent L/D response indicates carbon utilization by respiration via the succinate dehydrogenase complex in $\mathrm{D}$ and the inactivation or reversion of this process in $\mathrm{L}$. In contrast, the intermediates of the oxidative branch, such as citrate and aconitate, accumulated in $\mathrm{L}$ and reflected enhanced use of photosynthetically fixed organic carbon by ammonia assimilation via glutamine synthetase/glutamine:2-oxoglutarate aminotransferase (GS/GOGAT cycle). However, the amount of $2 \mathrm{OG}$ did not significantly differ between the L and D samples of all strains, ruling out the possibility that 2OG-mediated signaling was responsible for the observed expression changes in the CCM-related genes. The deficiency of the KaiAB1C1 system and the RpaA regulator did not affect the TCA pathways, but the utilization of carbon in the associated nitrogen assimilation is reported in the following section.

Nitrogen assimilation and amino acid biosynthesis. In addition to 2OG, the products of the GS/GOGAT cycle, i.e., glutamine and glutamate, and the subsequent aminotransferase product aspartate accumulate in illuminated wild-type cells (Figure 11). The accumulation of these amino acids paralleled the changes in the oxidative TCA branch. These concentration changes are consistent with the operation of ammonia assimilation in the L. Nitrogen $(\mathrm{N})$-assimilation requires the products of photosynthetic light reactions, such as ATP and reduced ferredoxin. Similar to the TCA pathways, the deficiency of KaiAB1C1 proteins did not affect ammonium assimilation or the associated glutamate/aspartate metabolism. The $\Delta r p a A$ mutant, however, differed in the L/D responses of glutamate but not of glutamine; specifically, the glutamate concentrations increased in D (Figure 11). This increase was associated with a significant accumulation of aspartate in the D and consistent with the supply directly from glutamate via the aspartate aminotransferase reaction, while the carbon backbone, oxaloacetate, may be supplied by phosphoenolpyruvate carboxylase (PEPC), malate dehydrogenase and the intermediates of the reductive TCA branch. The lack of a concomitant accumulation of $2 \mathrm{OG}$ indicated active reuse by the GS/GOGAT cycle. Upon illumination, the $\Delta r p a A$ cells overaccumulated Asp and $\mathrm{N}$-acetylglutamate, which is an additional direct product of glutamate in the pathway towards arginine biosynthesis (Figure 11). Collectively, our observations indicate overly active $\mathrm{N}$ assimilation in $\triangle r p a A$ cells that extends in $\mathrm{D}$ to multiple proteinogenic amino acid pools (Figure 5). Furthermore, in both diurnal phases, the amounts of nonproteinogenic amino acids, such as $\beta$-alanine and homoserine, increased, while nucleobases, such as thymine and uracil, increased in L (Figure 11, Supplemental Table S2). We hypothesize that the $\Delta r p a A$ mutant may not stop $\mathrm{N}$-assimilation during the night for an unknown reason.

Metabolic cofactors. The set of nucleotides and nucleosides monitored in the current study mostly exhibited minor pool changes that did not pass the significance threshold (Supplemental Table S1, Supplemental Table S2). The lack of significance in our study likely results from the high variation in the sampling time in the middle of the $\mathrm{L}$ phase. This phase still represents a time during which the concentrations of many nucleotides and nucleosides return from high levels in the morning (Saha et al., 2016) to the low levels present at the end of the day and during the night (Werner et al., 2019).

The levels of glutathione (GSH) and flavin mononucleotide (FMN) fluctuated less. Both metabolites indicate the deregulation of the redox state in $\triangle k a i A B l C l$ cells and greater deregulation in the $\triangle r p a A$ mutant. GSH failed to increase upon illumination in the $\triangle r p a A$ cells, whereas FMN accumulated constitutively in both the $\triangle k a i A B 1 C l$ and $\triangle r p a A$ cells.

Motivated by these observations, we monitored the nicotinamide adenine dinucleotide redox cofactors $\mathrm{NADP}^{+}, \mathrm{NADPH}, \mathrm{NAD}^{+}$and $\mathrm{NADH} . \mathrm{NADP}^{+}, \mathrm{NADPH}$ and $\mathrm{NAD}^{+}$were quantified, but these 
nucleotides and nucleosides did not pass our significance threshold (Supplemental Table S3). NADH was not detectable due to low concentrations. Our measurements indicated that $\mathrm{NADP}^{+}, \mathrm{NADPH}$ $\mathrm{NAD}^{+}$, and the NADPH/NADP ratio potentially increased in the wild type in the L phase compared to D. This increase in the NADPH/NADP ratio in L appeared to be absent from the $\triangle k a i A B 1 C 1$ and $\triangle r p a A$ mutant cells. In both mutants, this observation can be explained by an increase in the NADPH levels in the D (Supplemental Table S3).

\section{Discussion}

Based on metabolome analyses of Synechocystis mutants in L and D samples of autotrophically grown cultures, we can conclude that the Synechocystis KaiAB1C1-SasA-RpaA potential timing system largely affects the metabolic switch from photoautotrophy during the day to heterotrophy at night. This effect is comparable to the function of the Synechococcus sp. PCC 7942 circadian clock system, although the effects of the clock deficiency on the transcriptomic and metabolomic levels differ between these two cyanobacteria. These strain-specific differences are likely caused by differential adaptation of the two cyanobacteria. Facultative heterotrophy and the presence of alternative putative clock components in Synechocystis inevitably impact the transcriptional regulatory network with divergent metabolic consequences in the two cyanobacterial strains. Our findings suggest that the molecular mechanisms unraveled in one model organism may differ from those in other strains due to adaptive evolution. This assumption is consistent with findings suggesting that paralog duplications may quickly vanish from cyanobacterial genomes, e.g., of Acaryochloris or Chlorogloeopsis strains, unless they provide fitness benefits (Miller et al., 2011; Weissenbach et al., 2017). This reasoning likely applies to clock oscillator-related genes; for instance, the Nostoc linckia kaiABC gene family has been shown to respond to long-term differential stress by rapid adaptive radiation (Dvornyk et al., 2002).

In our Synechocystis model, the rpaA deletion had a much larger effect on metabolism than the kaiAB1Cl mutation, which is consistent with the more pronounced transcriptional changes in $\triangle r p a A$ (Köbler et al., 2018), although both mutants grew very similarly under these conditions. Most interestingly, $\triangle r p a A$ showed a remarkable overaccumulation of $2 \mathrm{PG}$ and amino acids in $\mathrm{D}$ with concurrent effects on the redox balance. The effects in the $\triangle$ kaiABlCl mutant are similar but smaller. This can be mainly explained by RpaA function in transcriptional regulation even in the absence of Kai-controlled phosphorylation. Phenotypic analyses of phosphor-mimicking variants of RpaA could be helpful to evaluate this hypothesis in future studies. Further, the lack of RpaA-RpaB interactions in the $\triangle r p a A$ mutant might affect the control of the large RpaB-dependent regulon, which partially overlaps with the RpaA regulon (e.g., control of the psaAB operon) (Seino et al., 2009; Köbler et al., 2018). Moreover, a recent study by Riediger et al. (2019) largely extended the RpaB regulon, which currently includes many enzymes involved in primary carbon and nitrogen metabolism, likely contributing to the larger impact of RpaA deficiency on L/D-mediated metabolic changes. In addition, or alternatively, the presence of the likely only partially neofunctionalized KaiB3C3 and KaiB2C2 systems in Synechocystis could compensate for the loss of the KaiAB1C1 complex. At least for the KaiC3-based system, it has been shown that it interacts with the KaiAB1C1 complex and modulates its function (Wiegard et al., 2020).

It has been suggested that the NADPH/NADP ratio plays a major role in the diurnal growth of Synechocystis (Saha et al., 2016). This ratio is known as the main redox signal and has a pronounced impact on enzyme activities in the $\mathrm{CBB}$ cycle, glycolysis and gluconeogenesis. For example, the NADPH/NADP ratio switches the activities of the CBB enzymes Gap2 and Prk on and off via the small redox-sensitive protein CP12 in L to D transitions (McFarlane et al., 2019). Hence, the tendency of higher NADPH/NADP ratios in the mutants, especially in $\triangle r p a A$, suggests that these and other 
metabolic redox cofactors likely contribute to the observed disbalanced carbon and nitrogen metabolism in L/D transitions. Moreover, the continued and enhanced activity of RubisCO in D leads to the observed accumulation of $2 \mathrm{PG}$ in both mutants due to the enhanced oxygenase reaction in the presence of lower $\mathrm{C}_{\mathrm{i}} / \mathrm{O}_{2}$ ratios in $\mathrm{D}$. $2 \mathrm{PG}$ can be toxic and provides a metabolic signal that falsely indicates carbon limitation. $2 \mathrm{PG}$ accumulation in $\triangle r p a A$ is much more pronounced than that in $\triangle k a i A B 1 C 1$, explaining the previously detected overexpression of transcripts involved in $\mathrm{C}_{\mathrm{i}}$ limitation response in $\triangle r p a A$ (Köbler et al., 2018). The PHB deficiency observed in both strains can be traced to the lower accumulation of mRNAs encoding the enzymes involved in PHB synthesis. Furthermore, this deficiency might be caused by the lower pyruvate pool and respective metabolic limitation of the acetyl-CoA precursor of PHB synthesis in the clock mutants. However, the lack of PHB should not affect the growth of cells in L/D cycles as shown by Damrow et al. (2016), suggesting that this deficiency is not the primary cause of the phenotype of the analyzed mutants.

Moreover, previous analyses showed that mixotrophic conditions, i.e., feeding glucose in the L, have an aggravating effect on the growth of both mutant strains (Dörrich et al., 2014; Köbler et al., 2018). In the present study, we provide evidence suggesting that high $\mathrm{CO}_{2}$ supplementation improves the ability of both mutants to grow at diurnal L/D cycles. In contrast, glucose addition and limiting $\mathrm{C}_{\mathrm{i}}$ availability result in redox imbalance. Therefore, the defect in the mutants' ability to grow diurnally is likely associated with disturbed redox homeostasis as previously shown in Synechococcus elongatus PCC 7942 (Diamond et al., 2017). The redox imbalance in the two mutants is also reflected by the transcriptional changes as genes encoding flavoproteins (Flv), especially Flv2 and Flv4, are significantly upregulated compared to those in the wild type (Köbler et al., 2018). These flavoproteins along with Flv1 and Flv3 are required for the Mehler-like reaction in Synechocystis to support growth under fluctuating or high light conditions (Santana-Sanchez et al., 2019).

Finally, motivated by the potential of Synechocystis and other cyanobacteria for biotechnological applications and the important role of the circadian clock in controlling cyanobacterial metabolism, several groups utilized deletion and overproduction strains of the SasA-RpaA output module and resulting analyzed metabolic changes. Most importantly, the overexpression of sasA and rpaA resulted in enhanced sugar catabolism in the D (Iijima et al., 2015; Osanai et al., 2015). Our analysis of a mutant lacking the rpaA gene reveals the opposite effects, i.e., more carbon is exported from the CBB cycle into lower glycolysis and TCA cycle, which is consistent with published data of rpaA overexpression (Iijima et al., 2015). Hence, the results presented here and by other groups provide evidence suggesting that an intact KaiAB1C1-based system is required in Synechocystis for the improved production of Cbased metabolites under natural L/D cycles with cyanobacteria.

\section{$5 \quad$ Author Contribution}

A.W., J.K., and C.K. designed research; N.M.S., Y.R., S.T, and C.K. performed research; N.M.S., Y.R., J.K., and M.H. analyzed the data; and J.K., M.H., A.W., N.M.S., and Y.R. wrote the paper.

\section{$6 \quad$ Funding}

The project was funded by grants of the research group (FOR2816) SCyCode "The AutotrophyHeterotrophy Switch in Cyanobacteria: Coherent Decision-Making at Multiple Regulatory Layers" to M.H. (HA 2002/23-1), A. W (WI 2014/10-1) and J.K. (KO 2329/7-1).

\section{$7 \quad$ Acknowledgment}


We thank Matthias Boll for providing the UPLC and Max Willistein for support in the operation of the UPLC. We further thank Judith Asal and Werner Bigott for excellent technical assistance.

\section{Conflict of Interest}

The authors declare that the research was conducted in the absence of any commercial or financial relationships that could be construed as a potential conflict of interest.

\section{$9 \quad$ References}

Beck, C., Hertel, S., Rediger, A., Lehmann, R., Wiegard, A., Kölsch, A., et al. (2014). Daily expression pattern of protein-encoding genes and small noncoding RNAs in Synechocystis sp. strain PCC 6803. Appl. Environ. Microbiol. 80, 5195-5206. doi:10.1128/AEM.01086-14.

Chen, X., Schreiber, K., Appel, J., Makowka, A., Fähnrich, B., Roettger, M., et al. (2016). The EntnerDoudoroff pathway is an overlooked glycolytic route in cyanobacteria and plants. Proc. Natl. Acad. Sci. 113, 5441-5446. doi:10.1073/pnas.1521916113.

Ciebiada, M., Kubiak, K., and Daroch, M. (2020). Modifying the cyanobacterial metabolism as a key to efficient biopolymer production in photosynthetic microorganisms. Int. J. Mol. Sci. 21, 7204. doi:10.3390/ijms21197204.

Cohen, S. E., and Golden, S. S. (2015). Circadian rhythms in Cyanobacteria. Microbiol. Mol. Biol. Rev. 79, 373-385. doi:10.1128/MMBR.00036-15.

Damrow, R., Maldener, I., and Zilliges, Y. (2016). The multiple functions of common microbial carbon polymers, glycogen and PHB, during stress responses in the non-diazotrophic cyanobacterium Synechocystis sp. PCC 6803. Front. Microbiol. 7, 966. doi:10.3389/fmicb.2016.00966.

Diamond, S., Rubin, B. E., Shultzaberger, R. K., Chen, Y., Barber, C. D., and Golden, S. S. (2017). Redox crisis underlies conditional light-dark lethality in cyanobacterial mutants that lack the circadian regulator, RpaA. Proc. Natl. Acad. Sci. 114, E580-E589. doi:10.1073/pnas.1613078114.

Dienst, D., Dühring, U., Mollenkopf, H.-J., Vogel, J., Golecki, J., Hess, W. R., et al. (2008). The cyanobacterial homologue of the RNA chaperone Hfq is essential for motility of Synechocystis sp. PCC 6803. Microbiology 154, 3134-3143. doi:10.1099/mic.0.2008/020222-0.

Dörrich, A. K., Mitschke, J., Siadat, O., and Wilde, A. (2014). Deletion of the Synechocystis sp. PCC 6803 kaiAB1C1 gene cluster causes impaired cell growth under light-dark conditions. Microbiology 160, 2538-2550. doi:10.1099/mic.0.081695-0.

Dvornyk, V., Vinogradova, O., and Nevo, E. (2002). Long-term microclimatic stress causes rapid adaptive radiation of kaiABC clock gene family in a cyanobacterium, Nostoc linckia, from "Evolution Canyons" I and II, Israel. Proc. Natl. Acad. Sci. 99, 2082-2087. doi:10.1073/pnas.261699498.

Eisenhut, M., Ruth, W., Haimovich, M., Bauwe, H., Kaplan, A., and Hagemann, M. (2008). The photorespiratory glycolate metabolism is essential for cyanobacteria and might have been conveyed endosymbiontically to plants. Proc. Natl. Acad. Sci. 105, 17199-17204. doi:10.1073/pnas.0807043105. 
Erban, A., Martinez-Seidel, F., Rajarathinam, Y., Dethloff, F., Orf, I., Fehrle, I., et al. (2020). "Multiplexed Profiling and Data Processing Methods to Identify Temperature-Regulated Using Gas Chromatography Coupled to Mass Spectrometry," in Hincha DK, Zuther E (eds) Plant cold acclimation: Methods in Molecular Biology (Methods and Protocols), 203-239. doi:10.1007/978-10716-0660-5_15.

Flügel, F., Timm, S., Arrivault, S., Florian, A., Stitt, M., Fernie, A. R., et al. (2017). The photorespiratory metabolite 2-phosphoglycolate regulates photosynthesis and starch accumulation in Arabidopsis. Plant Cell 29, 2537-2551. doi:10.1105/tpc.17.00256.

Gutu, A., and O'Shea, E. K. (2013). Two antagonistic clock-regulated histidine kinases time the activation of circadian gene expression. Mol. Cell 50, 288-294. doi:10.1016/j.molcel.2013.02.022.

Huege, J., Goetze, J., Schwarz, D., Bauwe, H., Hagemann, M., and Kopka, J. (2011). Modulation of the major paths of carbon in photorespiratory mutants of Synechocystis. PLoS One 6, e16278. doi:10.1371/journal.pone.0016278.

Iijima, H., Shirai, T., Okamoto, M., Kondo, A., Hirai, M. Y., and Osanai, T. (2015). Changes in primary metabolism under light and dark conditions in response to overproduction of a response regulator RpaA in the unicellular cyanobacterium Synechocystis sp. PCC 6803. Front. Microbiol. 6, 888. doi:10.3389/fmicb.2015.00888.

Ishiura, M., Kutsuna, S., Aoki, S., Iwasaki, H., Andersson, C. R., Tanabe, A., Golden, S. S., Johnson, C. H., and Kondo, T. (1998). Expression of a gene cluster kaiABC as a circadian feedback process in cyanobacteria. Science 281, 1519-1523. doi:10.1126/science.281.5382.1519.

Iwasaki, H., Taniguchi, Y., Ishiura, M., and Kondo, T. (1999). Physical interactions among circadian clock proteins KaiA, KaiB and KaiC in cyanobacteria. EMBO J. 18, 1137-1145. doi:10.1093/emboj/18.5.1137.

Jiang, Y.-L., Wang, X.-P., Sun, H., Han, S.-J., Li, W.-F., Cui, N., et al. (2018). Coordinating carbon and nitrogen metabolic signaling through the cyanobacterial global repressor NdhR. Proc. Natl. Acad. Sci. 115, 403-408. doi:10.1073/pnas.1716062115.

Kirsch, F., Klähn, S., and Hagemann, M. (2019). Salt-regulated accumulation of the compatible solutes sucrose and glucosylglycerol in cyanobacteria and its biotechnological potential. Front. Microbiol. 10, 2139. doi:10.3389/fmicb.2019.02139.

Köbler, C., Schultz, S.-J., Kopp, D., Voigt, K., and Wilde, A. (2018). The role of the Synechocystis sp. PCC 6803 homolog of the circadian clock output regulator RpaA in day-night transitions. Mol. Microbiol. 110, 847-861. doi:10.1111/mmi.14129.

Koch, M., Doello, S., Gutekunst, K., and Forchhammer, K. (2019). PHB is produced from glycogen turn-over during nitrogen starvation in Synechocystis sp. PCC 6803. Int. J. Mol. Sci. 20, 1942. doi:10.3390/ijms20081942.

Koch, M., Orthwein, T., Alford, J. T., and Forchhammer, K. (2020). The Slr0058 protein from Synechocystis sp. PCC 6803 is a novel regulatory protein involved in PHB granule formation. Front. Microbiol. 11, 809. doi:10.3389/fmicb.2020.00809. 
Kopka, J., Schauer, N., Krueger, S., Birkemeyer, C., Usadel, B., Bergmuller, E., et al. (2005). GMD@CSB.DB: the Golm Metabolome Database. Bioinformatics 21, 1635-1638. doi:10.1093/bioinformatics/bti236.

Kopka, J., Schmidt, S., Dethloff, F., Pade, N., Berendt, S., Schottkowski, M., et al. (2017). Systems analysis of ethanol production in the genetically engineered cyanobacterium Synechococcus sp. PCC 7002. Biotechnol. Biofuels 10, 56. doi:10.1186/s13068-017-0741-0.

Luedemann, A., Strassburg, K., Erban, A., and Kopka, J. (2008). TagFinder for the quantitative analysis of gas chromatography-mass spectrometry (GC-MS)-based metabolite profiling experiments. Bioinformatics 24, 732-737. doi:10.1093/bioinformatics/btn023.

Makowka, A., Nichelmann, L., Schulze, D., Spengler, K., Wittmann, C., Forchhammer, K., et al. (2020). Glycolytic shunts replenish the Calvin - Benson - Bassham Cycle as anaplerotic reactions in cyanobacteria. Mol. Plant 13, 471-482. doi:10.1016/j.molp.2020.02.002.

Markson, J. S., Piechura, J. R., Puszynska, A. M., and O’Shea, E. K. (2013). Circadian control of global gene expression by the cyanobacterial master regulator RpaA. Cell 155, 1396-1408. doi:10.1016/j.cell.2013.11.005.

McFarlane, C. R., Shah, N. R., Kabasakal, B. V., Echeverria, B., Cotton, C. A. R., Bubeck, D., et al. (2019). Structural basis of light-induced redox regulation in the Calvin-Benson cycle in cyanobacteria. Proc. Natl. Acad. Sci. 116, 20984-20990. doi:10.1073/pnas.1906722116.

Miller, S. R., Wood, A. M., Blankenship, R. E., Kim, M., and Ferriera, S. (2011). Dynamics of gene duplication in the genomes of chlorophyll $d$-producing cyanobacteria: Implications for the ecological niche. Genome Biol. Evol. 3, 601-613. doi:10.1093/gbe/evr060.

Nishimura, T., Takahashi, Y., Yamaguchi, O., Suzuki, H., Maeda, S., and Omata, T. (2008). Mechanism of low $\mathrm{CO}_{2}$-induced activation of the cmp bicarbonate transporter operon by a LysR family protein in the cyanobacterium Synechococcus elongatus strain PCC 7942. Mol. Microbiol. 68, 98-109. doi:10.1111/j.1365-2958.2008.06137.x.

Orf, I., Klähn, S., Schwarz, D., Frank, M., Hess, W. R., Hagemann, M., et al. (2015). Integrated analysis of engineered carbon limitation in a quadruple $\mathrm{CO}_{2} / \mathrm{HCO}_{3}$-uptake mutant of Synechocystis sp. PCC 6803. Plant Physiol. 169, 1787-1806. doi:10.1104/pp.15.01289.

Orthwein, T., Scholl, J., Spät, P., Lucius, S., Koch, M., Macek, B., et al. (2021). The novel PII interactor PirC identifies phosphoglycerate mutase as key control point of carbon storage metabolism in cyanobacteria. Proc. Natl. Acad. Sci. 118, e2019988118. doi:10.1073/pnas.2019988118.

Osanai, T., Shirai, T., Iijima, H., Kuwahara, A., Suzuki, I., Kondo, A., et al. (2015). Alteration of cyanobacterial sugar and amino acid metabolism by overexpression hik8, encoding a KaiC-associated histidine kinase. Environ. Microbiol. 17, 2430-2440. doi:10.1111/1462-2920.12715.

Pinto, F., Thapper, A., Sontheim, W., and Lindblad, P. (2009). Analysis of current and alternative phenol based RNA extraction methodologies for cyanobacteria. BMC Mol. Biol. 10, 79. doi:10.1186/1471-2199-10-79. 
Puszynska, A. M., and O'Shea, E. K. (2017). Switching of metabolic programs in response to light availability is an essential function of the cyanobacterial circadian output pathway. Elife 6:e23210. doi:10.7554/eLife. 23210 .

Rai, S., Lucius, S., Kern, R., Bauwe, H., Kaplan, A., Kopka, J., et al. (2018). The Synechocystis sp. PCC 6803 genome encodes up to four 2-phosphoglycolate phosphatases. Front. Plant Sci. 9, 1718. doi:10.3389/fpls.2018.01718.

Riediger, M., Kadowaki, T., Nagayama, R., Georg, J., Hihara, Y., and Hess, W. R. (2019). Biocomputational analyses and experimental validation identify the regulon controlled by the redoxresponsive transcription factor RpaB. iScience 15, 316-331. doi:10.1016/j.isci.2019.04.033.

Rippka, R., Deruelles, J., Herdman, M., Waterbury, J. B., and and Stanier, R. (1979). Generic assignments, strain histories and properties of pure cultures of cyanobacteria. Microbiology 111, 1-61.

Saha, R., Liu, D., Connor, A. H., Liberton, M., Yu, J., and Bhattacharyya-Pakrasi, M. (2016). Diurnal regulation of cellular processes in the cyanobacterium Synechocystis sp . strain PCC 6803: Insights from transcriptomic, fluxomic, and physiological analyses. 7, e00464-16. doi:10.1128/mBio.0046416.

Santana-Sanchez, A., Solymosi, D., Mustila, H., Bersanini, L., Aro, E.-M., and Allahverdiyeva, Y. (2019). Flavodiiron proteins 1-to-4 function in versatile combinations in $\mathrm{O}_{2}$ photoreduction in cyanobacteria. Elife 8:e45766. doi:10.7554/eLife.45766.

Seino, Y., Takahashi, T., and Hihara, Y. (2009). The response regulator RpaB binds to the upstream element of Photosystem I genes to work for positive regulation under low-light conditions in Synechocystis sp. Strain PCC 6803. J. Bacteriol. 191, 1581-1586. doi:10.1128/JB.01588-08.

Shibata, M., Katoh, H., Sonoda, M., Ohkawa, H., Shimoyama, M., Fukuzawa, H., et al. (2002). Genes essential to sodium-dependent bicarbonate transport in cyanobacteria. J. Biol. Chem. 277, 1865818664. doi:10.1074/jbc.M112468200.

Shibata, M., Ohkawa, H., Kaneko, T., Fukuzawa, H., Tabata, S., Kaplan, A., et al. (2001). Distinct constitutive and low- $\mathrm{CO}_{2}$-induced $\mathrm{CO}_{2}$ uptake systems in cyanobacteria: Genes involved and their phylogenetic relationship with homologous genes in other organisms. Proc. Natl. Acad. Sci. 98, 1178911794. doi:10.1073/pnas.191258298.

Tamoi, M., Miyazaki, T., Fukamizo, T., and Shigeoka, S. (2005). The Calvin cycle in cyanobacteria is regulated by $\mathrm{CP} 12$ via the $\mathrm{NAD}(\mathrm{H}) / \mathrm{NADP}(\mathrm{H})$ ratio under light/dark conditions. Plant J. 42, 504-513. doi:10.1111/j.1365-313X.2005.02391.x.

Taroncher-Oldenburg, G., Nishina, K., and Stephanopoulos, G. (2000). Identification and analysis of the polyhydroxyalkanoate-specific $\beta$-ketothiolase and acetoacetyl coenzyme A reductase genes in the cyanobacterium Synechocystis sp. strain PCC6803. Appl. Environ. Microbiol. 66, 4440-4448. doi:10.1128/AEM.66.10.4440-4448.2000.

Trautmann, D., Voß, B., Wilde, A., Al-Babili, S., and Hess, W. R. (2012). Microevolution in cyanobacteria: Re-sequencing a motile substrain of Synechocystis sp. PCC 6803. DNA Res. 19, 435448. doi:10.1093/dnares/dss024. 
Weissenbach, J., Ilhan, J., Bogumil, D., Hülter, N., Stucken, K., and Dagan, T. (2017). Evolution of chaperonin gene duplication in Stigonematalean Cyanobacteria (Subsection V). Genome Biol. Evol. 9, evw287. doi:10.1093/gbe/evw287.

Werner, A., Broeckling, C. D., Prasad, A., and Peebles, C. A. M. (2019). A comprehensive time-course metabolite profiling of the model cyanobacterium Synechocystis sp. PCC 6803 under diurnal light:dark cycles. Plant J. 99, 379-388. doi:10.1111/tpj.14320.

Wiegard, A., Dörrich, A. K., Deinzer, H.-T., Beck, C., Wilde, A., Holtzendorff, J., et al. (2013). Biochemical analysis of three putative KaiC clock proteins from Synechocystis sp. PCC 6803 suggests their functional divergence. Microbiology 159, 948-958. doi:10.1099/mic.0.065425-0.

Wiegard, A., Köbler, C., Oyama, K., Dörrich, A. K., Azai, C., Terauchi, K., et al. (2020). Synechocystis KaiC3 displays temperature- and KaiB-dependent ATPase activity and is important for growth in darkness. J. Bacteriol. 202, e00478-19. doi:10.1128/JB.00478-19.

Xu, M., Bernát, G., Singh, A., Mi, H., Rögner, M., Pakrasi, H. B., et al. (2008). Properties of mutants of Synechocystis sp. strain PCC 6803 lacking inorganic carbon sequestration systems. Plant Cell Physiol. 49, 1672-1677. doi:10.1093/pcp/pcn139.

Zhang, Y., Krahnert, I., Bolze, A., Gibon, Y., and Fernie, A. R. (2020). Adenine nucleotide and nicotinamide adenine dinucleotide measurements in plants. Curr. Protoc. Plant Biol. 5, e20115. doi:10.1002/cppb.20115. 


\section{$10 \quad$ Figure legends}

Figure 1. Model of the Synechocystis Kai system. In Synechocystis, the KaiAB1C1 system is the closest homolog to the well-known circadian clock system from Synechococcus elongatus PCC 7942. The output signaling response of KaiC1 relies on the SasA-RpaA two-component system. The transcription factor RpaA controls gene expression in L and D conditions. RpaA can interact with other regulators (e.g Pix and RpaB) and partially targets the same genes as the transcription factor RpaB. The KaiAB1C1 complex is intertwined with the nonstandard KaiB3C3 system in Synechocystis, but the direction of signaling between the two systems remains unclear. The red circles represent phosphorylation sites.

Figure 2. Growth of Synechocystis mutants. Wild type (WT), $\triangle r p a A$ and $\triangle$ kaiABlCl deletion mutants were grown in a 12-hour L/12-hour D cycle with ambient air (A) and $1 \% \mathrm{CO}_{2}(\mathbf{B})$. The light intensity during cultivation was $75 \mu \mathrm{mol}$ photons $\mathrm{m}^{-2} \mathrm{~s}^{-1}$, which was increased to $150 \mu \mathrm{mol}$ photons $\mathrm{m}^{-}$ ${ }^{2} \mathrm{~s}^{-1}$ in $\mathbf{B}$ after the cultures reached an OD $750 \mathrm{~nm}$ of $\sim 1$. Each point represents the mean of three biological replicates ( \pm standard deviation).

Figure 3. The $\triangle r p a A$ and $\triangle k a i A B 1 C 1$ strains show lower $\mathrm{PHB}$ accumulation at high $\mathrm{CO}_{2}$ concentrations. PHB was quantified in cells cultivated in a 12-hour L/12-hour D cycle and $1 \% \mathrm{CO}_{2}$. The samples used for the PHB measurements were collected in the stationary phase at an OD $750 \mathrm{~nm}$ of $\sim 7$ in the $\mathrm{L}$ and $\mathrm{D}$ phases of the $7^{\text {th }} \mathrm{L} / \mathrm{D}$ cycle one hour before switching. Each bar displays the mean of three technical replicates ( \pm standard deviation).

Figure 4. Global analysis of the metabolic L/D switch in the $\triangle k a i A B 1 C 1$ and $\triangle r p a A$ mutants. (A) Design of the two-factorial entrainment experiment. Wild-type and mutant cells, i.e., the first experimental factor (genotype), were precultivated under continuous light, divided into independent cultures of $\mathrm{OD}_{750 \mathrm{~nm}}=0.6$ in ambient air, and sampled in the middle of the first night (D phase) and first day ( $\mathrm{L}$ phase) after entrainment, i.e., the second experimental factor (illumination). Three independent replicate cultures in each phase were analyzed by a quantitative targeted LC-MS analysis and nontargeted GC-MS metabolome profiling of the primary metabolome. (B) Hierarchical cluster analysis (HCA) of mutant and wild-type metabolomes. Annotated LC- and GC-MS metabolite data separately maximum scaled and then combined, log-transformed, autoscaled, and clustered using Pearson's correlation distance metric and average linkage. HCA includes a scale of the node height and bootstrap-support analysis by 1000 iterations. The values represent the 'approximately unbiased' bootstrap probability, and in brackets, the conventional bootstrap probability obtained using the 'pvclust' $\mathrm{R}$ package is shown. Values of 100 represent the highest node probability. (C) Principal component analysis (PCA) of the same data. PC1 and PC3 demonstrate the variance contribution of illumination (PC1) and the $\triangle r p a A$ samples collected in the D phase (PC3) to the metabolomics data set. Yellow arrows represent the score biplot of the samples. The gray underlay indicates the loading contributions of the metabolites. A corresponding supplement contains a biplot of PC1 and PC2 and a scree plot of the variance represented by the top 10 PCs (Supplemental Figure S3).

Figure 5. Relative abundances and fold changes (FC) of metabolite concentrations in the $L$ phase compared to the $D$ phase. The figure includes identified metabolites that exhibited significant changes in $\mathrm{L}$ metabolism compared to $\mathrm{D}$ metabolism (Tukey test $\mathrm{P}<0.05$ ). Fold changes are color-coded as follows: significant increases, red $(>2-\mathrm{FC})$, light red $(<2-\mathrm{FC})$, significant decreases, blue $(<0.5-\mathrm{FC})$, and light blue $(>0.5-\mathrm{FC})$; the complete numerical data are provided in Supplemental Table S1. The grouping of the metabolites into the L/D response groups (L/D groups) was performed according to the changes in the wild type (L/D group I-II) and considered inverted (L/D group III-IV) changes in 
the mutants or emergent changes (L/D group V). Heat map: light yellow, low abundance; dark yellow, high abundance; gray, undetectable.

Figure 6. Summary of the metabolic changes in the mutants of Synechocystis. Venn diagram analysis of all significant (Tukey test, $P<0.05$ ) metabolic changes among the set of identified and yet unidentified metabolites. Intersections show significant changes in the metabolite pools shared between the $\triangle$ kaiABlCl (brown) and $\triangle r p a A$ (blue) mutants. Zoom-ins show the contribution of the two diurnal phases to the respective sets, D phase (gray) and L phase (yellow). Note the large overlap between the mutants and the more frequent specific responses of the $\Delta r p a A$ cells.

Figure 7. Photorespiration. Box plots showing the maximum normalized relative changes in the metabolite concentrations and Tukey test results $(P<0.05, \mathrm{n}=3)$. Metabolite levels that significantly differ are indicated by lowercase letters and color coding. Note the moderate and strong increases in the RubisCO products 3PGA and 2PG in the $\triangle$ kaiABlC1 mutant and the $\triangle r p a A$ mutant, respectively. High 2PG accumulation in the $\triangle r p a A$ mutant continues in the D phase and is associated with glycolate accumulation in the light phase. Serine and glycine respond inversely to RpaA deficiency. Glycine fails to accumulate in the $\mathrm{D}$, while serine accumulates in the L. Dashed arrows indicate multiple reaction steps. Relevant enzymes and complexes include (gray) glycolate-2P phosphatase (Pgp), glycolate dehydrogenase (Glydh), aminotransferase (At), glycine decarboxylase complex (Gdc), serine hydroxymethyltransferase (Shmt), tetrahydrofolate (THF), hydroxypyruvate reductase (Hpr), and glycerate kinase $(\mathrm{Gk})$.

Figure 8. Increased RubisCO abundance in mutants compared to wild type (WT). Comparison of the RubisCO levels via immunoblot analysis using anti-RbcL. 10 or $15 \mu \mathrm{g}$ of crude cell extract were used for SDS-PAGE. The samples were collected 5.5 h after the transition to D or L as shown in Figure $4 \mathrm{~A}$.

Figure 9. Lower glycolysis, glutamate, and the pyruvate family of amino acids. Box plots showing the maximum normalized relative changes in the metabolite concentrations and Tukey test results $(P$ $<0.05, \mathrm{n}=3$ ). Metabolite levels that significantly differ are indicated by lowercase letters and color coding. Note the subtle changes in the $\triangle$ kaiABlCl mutant, while the $\triangle$ rpaA mutant exhibited significantly increased pyruvate family amino acids, 3PGA, PEP and glutamate in D. These changes are accompanied by pyruvate depletion in the $\mathrm{D}$ phase relative to the wild type. In the L, 3PGA accumulates in the $\triangle$ kaiABlCl mutant relative to the wild type. In the $\triangle r p a A$ mutant, 3PGA accumulates to even higher levels, and the 2PGA and PEP increases become significant. Dashed arrows indicate multiple reaction steps. Relevant enzymes and complexes include (gray) ribulose-1,5bisphosphate carboxylase-oxygenase (RubisCO), phosphoglycerate mutase (Pgam), enolase (Eno), phosphoenolpyruvate synthase (Peps), pyruvate kinase $(\mathrm{Pk})$, pyruvate dehydrogenase (Pdh), alanine dehydrogenase (Aladh), and alanine transaminase (Alat).

Figure 10. Tricarboxylic acid pathways. Box plots showing the maximum normalized relative changes in the metabolite concentrations and Tukey test results $(P<0.05, \mathrm{n}=3)$. Metabolite levels that significantly differ are indicated by lowercase letters and color coding. Note the inverse accumulation of metabolites from the oxidative and reductive branches of the TCA pathways. The concentrations of the intermediates of the TCA reactions were largely unchanged in the $\triangle$ kaiABlCl and $\triangle r p a A$ cells. Dashed arrows indicate multiple reaction steps. Relevant enzymes and complexes included (gray) phosphoenolpyruvate synthase (PepS), pyruvate kinase $(\mathrm{Pk})$, pyruvate dehydrogenase (Pdh), phosphoenolpyruvate carboxylase (Pepc), citrate synthase (Cs), aconitase (Acn), isocitrate 
dehydrogenase (Idh), succinate semialdehyde dehydrogenase (Ssadh), succinate dehydrogenase (Sdh), fumarase $(\mathrm{Fh})$, malate dehydrogenase $(\mathrm{Mdh})$, and malic enzyme $(\mathrm{Me})$.

Figure 11. Nitrogen assimilation and associated amino acid biosynthesis. Box plots showing the maximum normalized relative changes in the metabolite concentrations and Tukey test results $(P<$ $0.05, \mathrm{n}=3$ ). Metabolite levels that significantly differ are indicated by lowercase letters and color coding. Note the increase in aspartate and the D accumulation of glutamate (Figure 11) in the $\triangle r p a A$ mutant. Relevant enzymes and complexes include (gray) glutamine synthetase (Gs), glutamine 2oxoglutarate aminotransferase (Gogat), aspartate aminotransferase (Aspat), asparagine synthetase (As), aspartate decarboxylase (Aspdc), and glutamate $\mathrm{N}$-acetyltransferase (Glunact). 


\section{Figures}

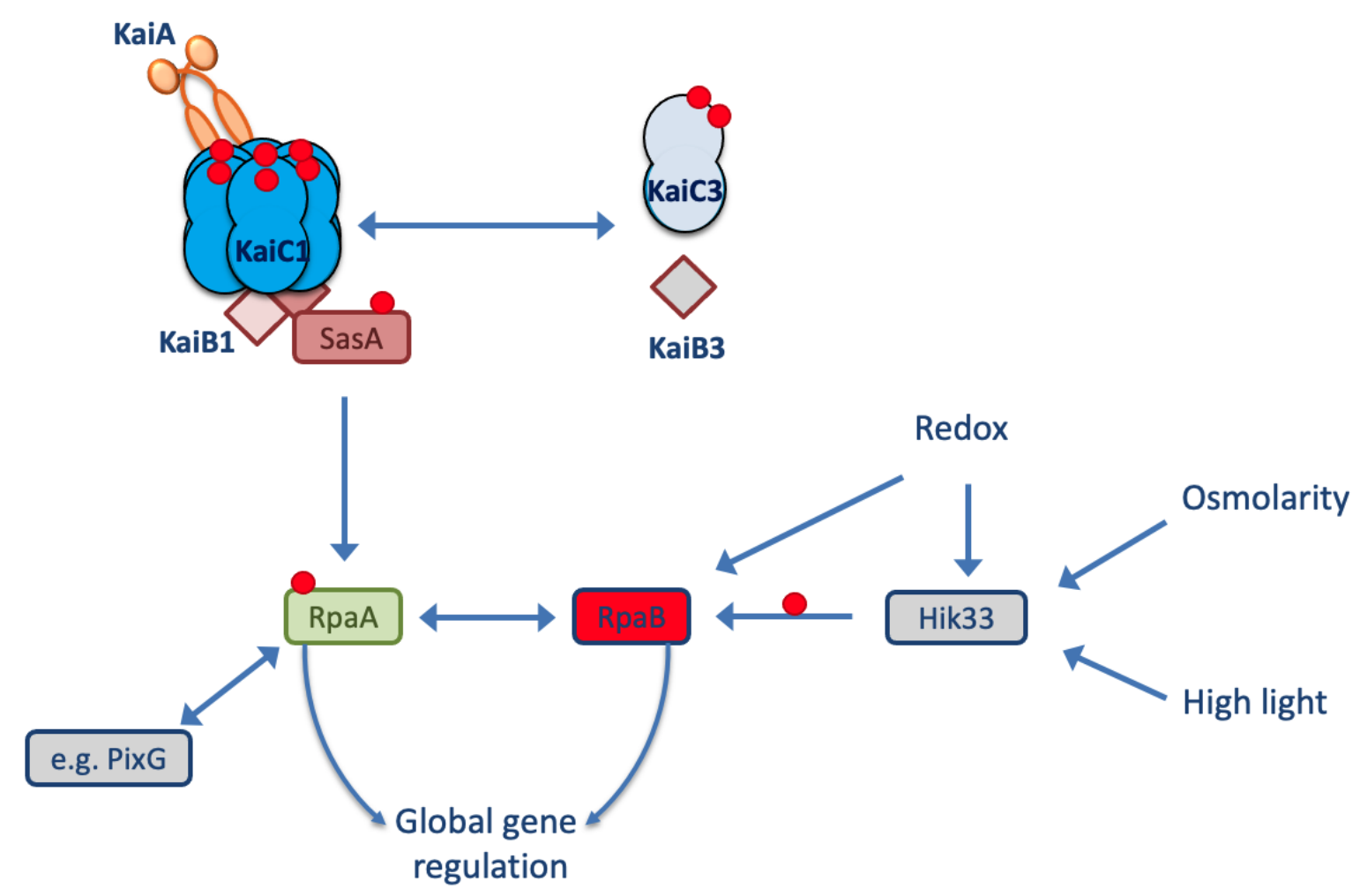

Figure 1. Model of the Synechocystis Kai system. In Synechocystis, the KaiAB1C1 system is the closest homolog to the well-known circadian clock system from Synechococcus elongatus PCC 7942. The output signaling response of KaiC1 relies on the SasA-RpaA two-component system. The transcription factor RpaA controls gene expression in L and D conditions. RpaA can interact with other regulators (e.g Pix and $\mathrm{RpaB}$ ) and partially targets the same genes as the transcription factor RpaB. The KaiAB1C1 complex is intertwined with the nonstandard KaiB3C3 system in Synechocystis, but the direction of signaling between the two systems remains unclear. The red circles represent phosphorylation sites. 

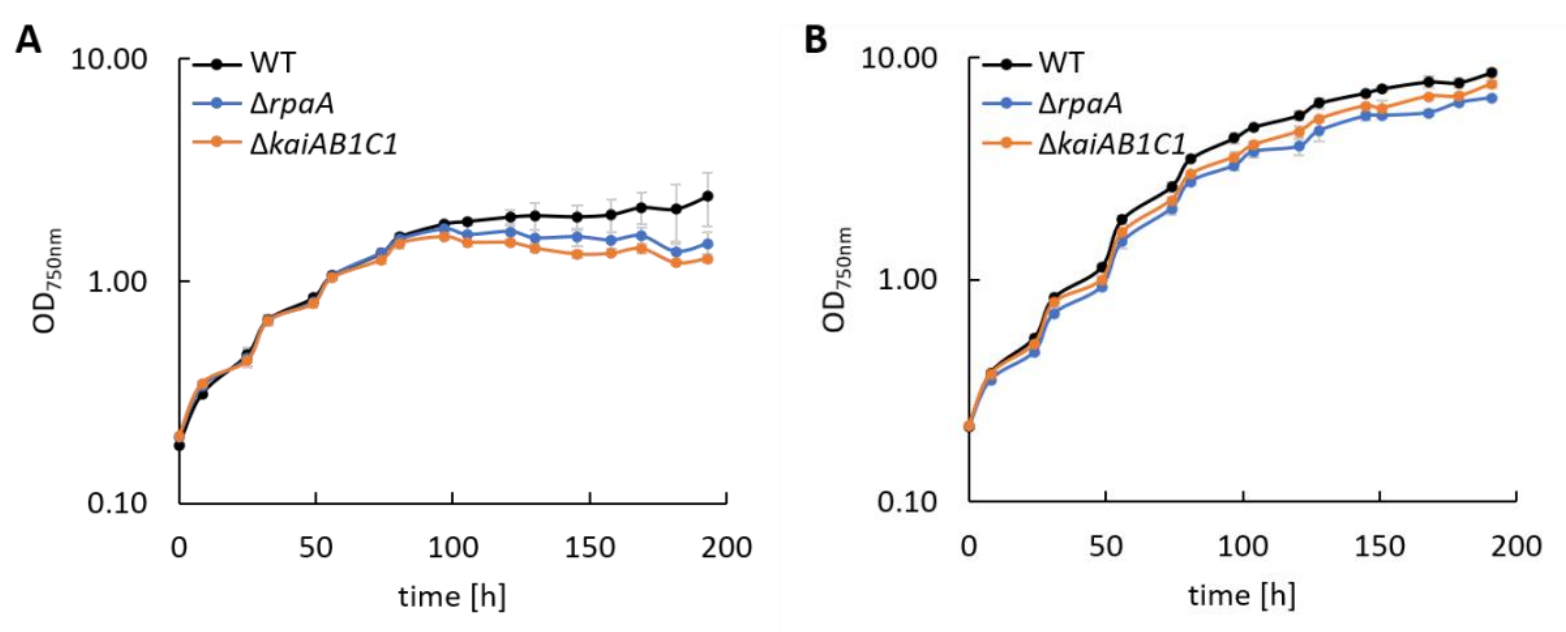

Figure 2. Growth of Synechocystis mutants. Wild type (WT), $\triangle r p a A$ and $\triangle k a i A B 1 C 1$ deletion mutants were grown in a 12-hour L/12-hour D cycle with ambient air (A) and $1 \% \mathrm{CO}_{2}$ (B). The light intensity during cultivation was $75 \mu \mathrm{mol}$ photons $\mathrm{m}^{-2} \mathrm{~s}^{-1}$, which was increased to $150 \mu \mathrm{mol}$ photons $\mathrm{m}^{-}$ ${ }^{2} \mathrm{~s}^{-1}$ in $\mathbf{B}$ after the cultures reached an OD $750 \mathrm{~nm}$ of $\sim 1$. Each point represents the mean of three biological replicates ( \pm standard deviation). 


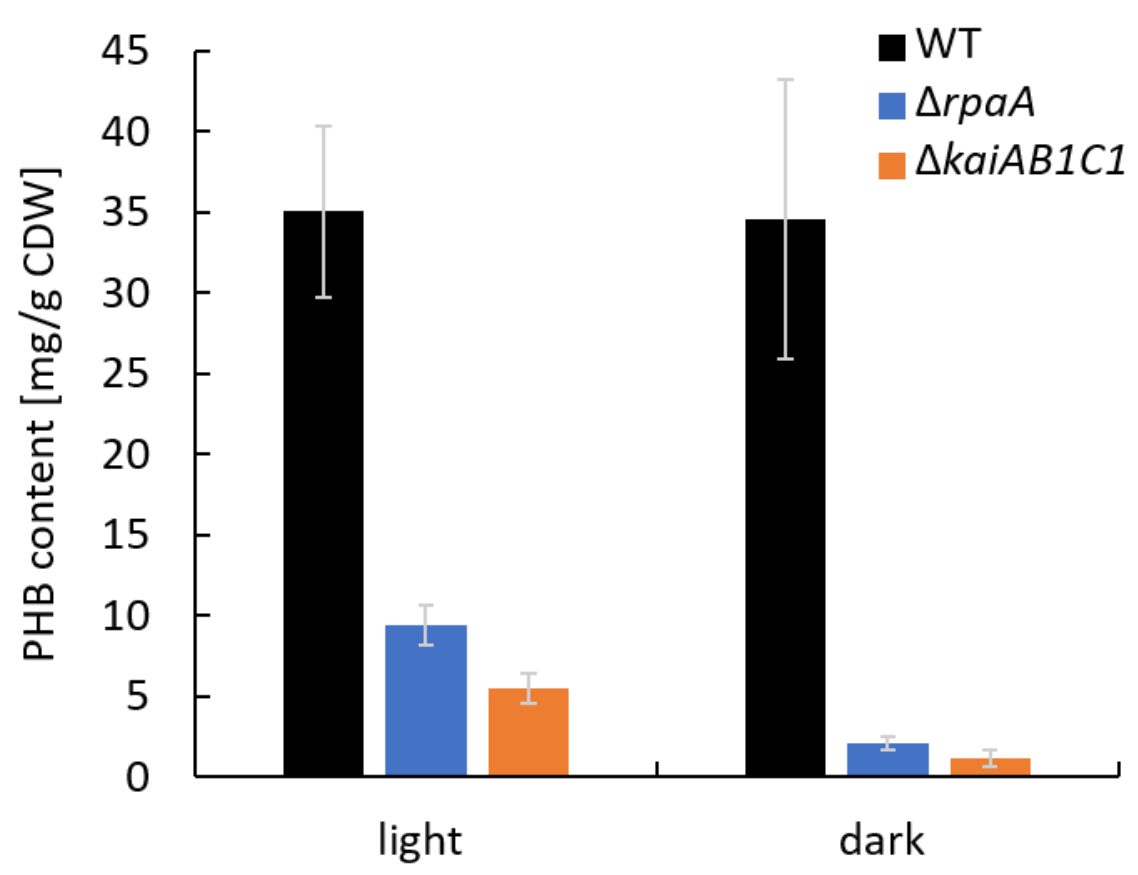

Figure 3. The $\triangle r p a A$ and $\triangle k a i A B 1 C 1$ strains show lower $\mathrm{PHB}$ accumulation at high $\mathrm{CO}_{2}$ concentrations. PHB was quantified in cells cultivated in a 12-hour L/12-hour D cycle and $1 \% \mathrm{CO}_{2}$. The samples used for the PHB measurements were collected in the stationary phase at an $\mathrm{OD}_{750} \mathrm{~nm}$ of $\sim 7$ in the $\mathrm{L}$ and $\mathrm{D}$ phases of the $7^{\text {th }} \mathrm{L} / \mathrm{D}$ cycle one hour before switching. Each bar displays the mean of three technical replicates ( \pm standard deviation). 


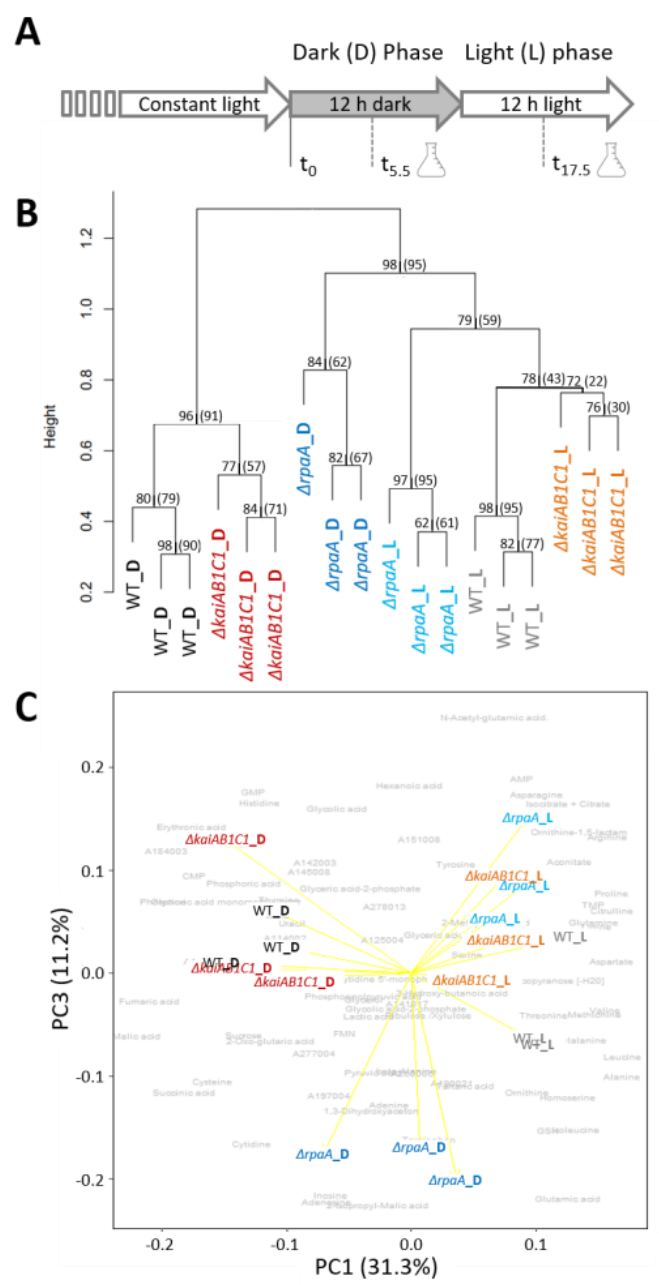

Figure 4. Global analysis of the metabolic L/D switch in the $\triangle k a i A B 1 C 1$ and $\Delta r p a A$ mutants. (A) Design of the two-factorial entrainment experiment. Wild-type and mutant cells, i.e., the first experimental factor (genotype), were precultivated under continuous light, divided into independent cultures of $\mathrm{OD}_{750 \mathrm{~nm}}=0.6$ in ambient air, and sampled in the middle of the first night (D phase) and first day (L phase) after entrainment, i.e., the second experimental factor (illumination). Three independent replicate cultures in each phase were analyzed by a quantitative targeted LC-MS analysis and nontargeted GC-MS metabolome profiling of the primary metabolome. (B) Hierarchical cluster analysis (HCA) of mutant and wild-type metabolomes. Annotated LC- and GC-MS metabolite data separately maximum scaled and then combined, log-transformed, autoscaled, and clustered using Pearson's correlation distance metric and average linkage. HCA includes a scale of the node height and bootstrap-support analysis by 1000 iterations. The values represent the 'approximately unbiased' bootstrap probability, and in brackets, the conventional bootstrap probability obtained using the 'pvclust' R package is shown. Values of 100 represent the highest node probability. (C) Principal component analysis (PCA) of the same data. PC1 and PC3 demonstrate the variance contribution of illumination (PC1) and the $\triangle r p a A$ samples collected in the D phase (PC3) to the metabolomics data set. Yellow arrows represent the score biplot of the samples. The gray underlay indicates the loading contributions of the metabolites. A corresponding supplement contains a biplot of PC1 and PC2 and a scree plot of the variance represented by the top 10 PCs (Supplemental Figure S3). 


\begin{tabular}{|c|c|c|c|c|c|c|}
\hline Metabolite & Technology & Relative & e Abundance & & $\begin{array}{l}\text { FC } \\
\text { ht } /\end{array}$ & \\
\hline & & 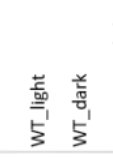 & 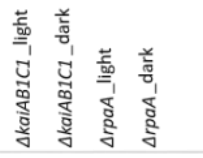 & $\xi$ & $\begin{array}{l}\tilde{J} \\
\overline{0} \\
\bar{v} \\
\bar{v}\end{array}$ & $\begin{array}{l}\text { d } \\
\text { g }\end{array}$ \\
\hline L/D-group I & & & & & & \\
\hline Glycolic acid-2-phosphate & GC-MS & $0.14-0$ & $\begin{array}{llll}0.30 & 0.32 & 0.79 & 0.74\end{array}$ & + & ns & ns \\
\hline Glucopyranose [-H20] & GC-MS & 0.810 .230 & 0.400 .400 .540 .39 & + & ns & ns \\
\hline Threonine & LC-MS & 0.770 .550 & 0.810 .740 .950 .87 & + & ns & ns \\
\hline Alanine & LC-MS & 0.820 .200 & 0.930 .260 .590 .61 & + & + & ns \\
\hline Valine & LC-MS & $0.580 .16 \mathrm{c}$ & $0.680 .28 \quad 0.830 .53$ & + & + & ns \\
\hline Isoleucine & LC-MS & $0.390 .13 \mathrm{c}-\mathrm{C}-\mathrm{l}$ & 0.450 .210 .600 .82 & + & + & ns \\
\hline Leucine & LC-MS & 0.700 .110 & 0.640 .190 .890 .63 & + & + & ns \\
\hline Methionine & LC-MS & $0.830 .16 c$ & 0.760 .330 .690 .49 & + & + & ns \\
\hline Homoserine & GC-MS & $0.230 .08 \mathrm{C}$ & 0.670 .090 .680 .76 & + & + & ns \\
\hline Phenylalanine & LC-MS & 0.440 .170 & 0.620 .300 .790 .64 & + & + & ns \\
\hline Citrulline & LC-MS & 0.400 .060 & 0.530 .120 .480 .15 & + & + & ns \\
\hline GSH & LC-MS & 0.890 .530 & 0.960 .490 .570 .80 & + & + & ns \\
\hline Glyceric acid-3-phosphate & LC-MS & $0.140 .06 \mathrm{C}$ & $\begin{array}{llll}0.35 & 0.24 & 0.82 & 0.19\end{array}$ & + & ns & + \\
\hline Aconitate & LC-MS & $0.890 .50 \mathrm{c}$ & $\begin{array}{llll}0.60 & 0.53 & 0.75 & 0.42\end{array}$ & + & ns & + \\
\hline Arginine & LC-MS & $0.360 .08 \mathrm{c}$ & 0.260 .080 .650 .09 & + & + & + \\
\hline Aspartate & LC-MS & 0.340 .120 & 0.400 .080 .910 .31 & + & + & + \\
\hline Proline & LC-MS & 0.290 .050 & 0.410 .060 .680 .09 & + & + & + \\
\hline L/D-group II & & & & & & \\
\hline Sucrose & GC-MS & $0.010 .43 c$ & 0.030 .040 .080 .08 & - & ns & ns \\
\hline Glycine & LC-MS & $0.330 .89 \mathrm{c}$ & 0.470 .750 .360 .41 & - & ns & ns \\
\hline Phosphoric acid monomethyl ester & GC-MS & 0.370 .760 & 0.740 .900 .690 .67 & - & ns & ns \\
\hline Erythronic acid & GC-MS & 0.530 .810 & 0.660 .850 .690 .59 & - & ns & ns \\
\hline Thymine & GC-MS & $0.500 .84 \mathrm{C}$ & $\begin{array}{l}0.780 .770 .890 .81 \\
\end{array}$ & - & ns & ns \\
\hline Lactic acid & GC-MS & -0.440 & 0.070 .050 .090 .09 & - & ns & ns \\
\hline Tartaric acid & GC-MS & -0.300 & 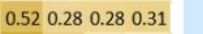 & - & ns & ns \\
\hline Hexanoic acid & GC-MS & - 0.250 & 0.710 .360 .140 .06 & - & ns & ns \\
\hline Glycerol & GC-MS & - 0.410 & 0.350 .070 .020 .14 & . & ns & ns \\
\hline Fumaric acid & GC-MS & $0.170 .55 \mathrm{c}$ & 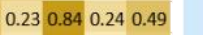 & - & - & ns \\
\hline Malic acid & GC-MS & $0.110 .56 \mathrm{c}$ & 0.140 .860 .110 .42 & - & - & - \\
\hline L/D-group III & & & & & & \\
\hline Glutamic acid & GC-MS & $0.280 .06 \mathrm{C}$ & 0.320 .030 .270 .76 & + & + & - \\
\hline L/D-group IV & & & & & & \\
\hline Pyruvic acid & GC-MS & -0.700 & 0.500 .410 .20 - & - & ns & + \\
\hline Histidine & LC-MS & $0.260 .58 \mathrm{c}$ & 0.440 .800 .770 .40 & - & ns & + \\
\hline L/D-group V & & & & & & \\
\hline Glutamine & LC-MS & $0.240 .14 \mathrm{C}$ & $0.280 .03 \quad 0.480 .13$ & ns & + & ns \\
\hline Lysine & LC-MS & $0.280 .13 \mathrm{c}$ & 0.280 .020 .470 .11 & ns & + & ns \\
\hline Ornithine-1,5-lactam & GC-MS & $0.120 .05 \mathrm{c}$ & 0.230 .040 .500 .07 & ns & + & + \\
\hline N-Acetyl-glutamic acid. & GC-MS & $0.290 .26 c$ & 0.400 .130 .630 .07 & ns & ns & + \\
\hline Isocitrate + Citrate & LC-MS & $0.850 .58 \mathrm{c}$ & 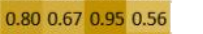 & ns & ns & + \\
\hline TMP & LC-MS & 0.850 .680 & 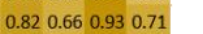 & ns & ns & + \\
\hline CMP & LC-MS & 0.530 .770 & 0.600 .900 .700 .67 & ns & - & ns \\
\hline & & not & $t$ detected $(-)$ & & gnifica & \\
\hline
\end{tabular}

Figure 5. Relative abundances and fold changes (FC) of metabolite concentrations in the $\mathrm{L}$ phase compared to the $\mathbf{D}$ phase. The figure includes identified metabolites that exhibited significant changes in $\mathrm{L}$ metabolism compared to $\mathrm{D}$ metabolism (Tukey test $\mathrm{P}<0.05$ ). Fold changes are color-coded as follows: significant increases, red $(>2-\mathrm{FC})$, light red $(<2-\mathrm{FC})$, significant decreases, blue $(<0.5-\mathrm{FC})$, and light blue $(>0.5-\mathrm{FC})$; the complete numerical data are provided in Supplemental Table S1. The grouping of the metabolites into the L/D response groups (L/D groups) was performed according to the changes in the wild type (L/D group I-II) and considered inverted (L/D group III-IV) changes in the mutants or emergent changes (L/D group V). Heat map: light yellow, low abundance; dark yellow, high abundance; gray, undetectable. 


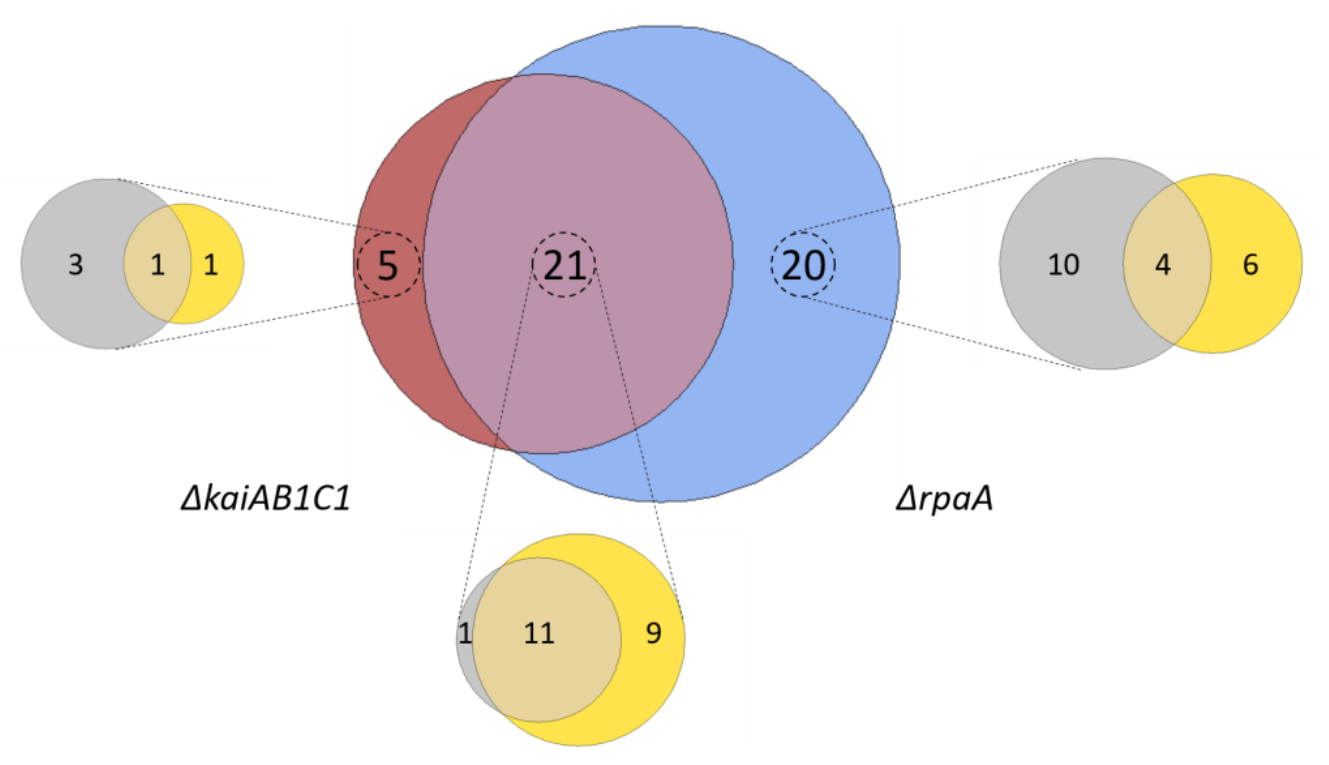

Figure 6. Summary of the metabolic changes in the mutants of Synechocystis. Venn diagram analysis of all significant (Tukey test, $P<0.05$ ) metabolic changes among the set of identified and yet unidentified metabolites. Intersections show significant changes in the metabolite pools shared between the $\triangle k a i A B 1 C 1$ (brown) and $\triangle r p a A$ (blue) mutants. Zoom-ins show the contribution of the two diurnal phases to the respective sets, D phase (gray) and L phase (yellow). Note the large overlap between the mutants and the more frequent specific responses of the $\triangle r p a A$ cells. 


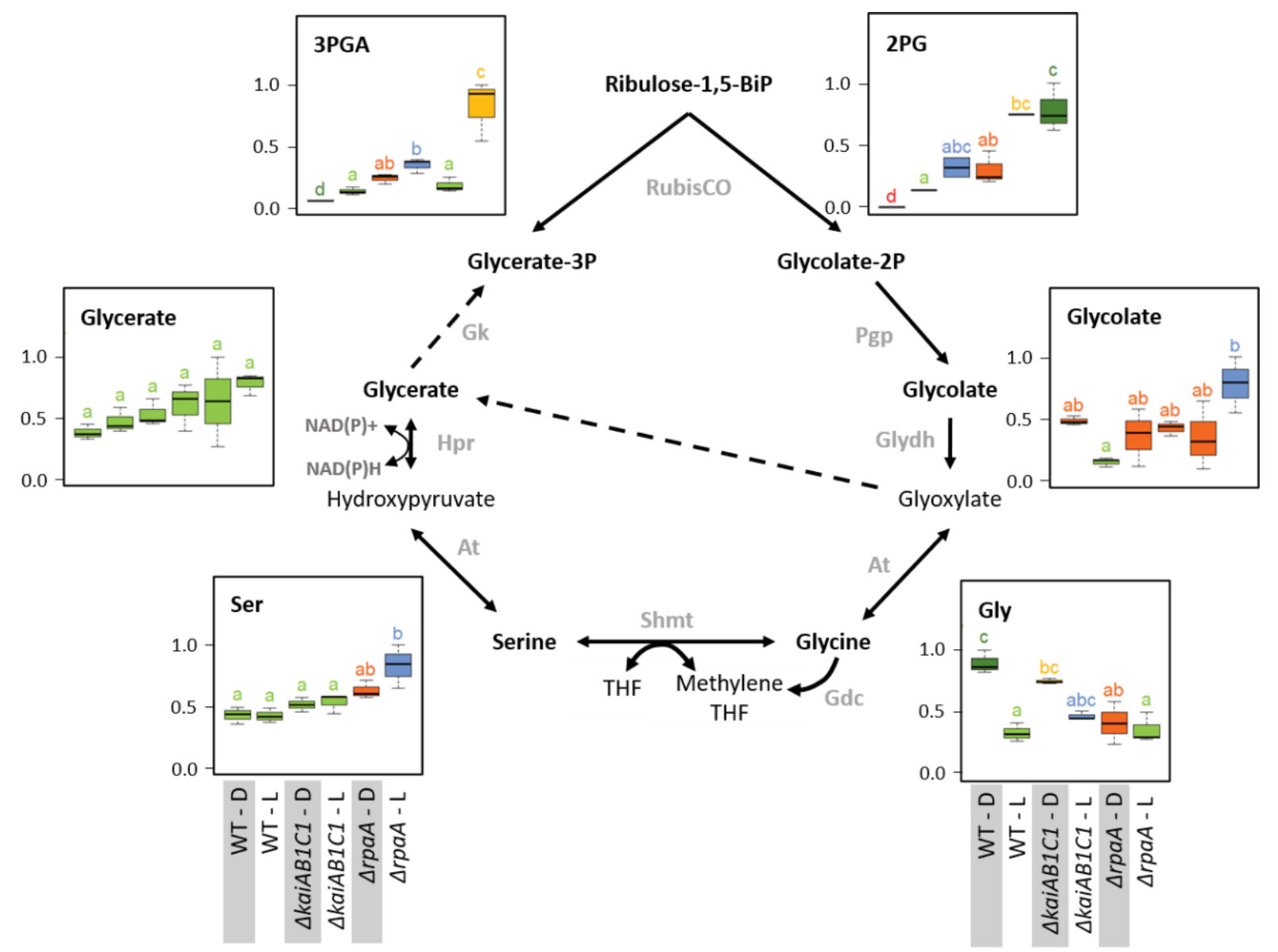

Figure 7. Photorespiration. Box plots showing the maximum normalized relative changes in the metabolite concentrations and Tukey test results $(P<0.05, \mathrm{n}=3)$. Metabolite levels that significantly differ are indicated by lowercase letters and color coding. Note the moderate and strong increases in the RubisCO products 3PGA and 2PG in the $\triangle$ kaiAB1C1 mutant and the $\triangle r p a A$ mutant, respectively. High 2PG accumulation in the $\triangle r p a A$ mutant continues in the D phase and is associated with glycolate accumulation in the light phase. Serine and glycine respond inversely to RpaA deficiency. Glycine fails to accumulate in the $\mathrm{D}$, while serine accumulates in the L. Dashed arrows indicate multiple reaction steps. Relevant enzymes and complexes include (gray) glycolate-2P phosphatase (Pgp), glycolate dehydrogenase (Glydh), aminotransferase (At), glycine decarboxylase complex (Gdc), serine hydroxymethyltransferase (Shmt), tetrahydrofolate (THF), hydroxypyruvate reductase (Hpr), and glycerate kinase (Gk). 


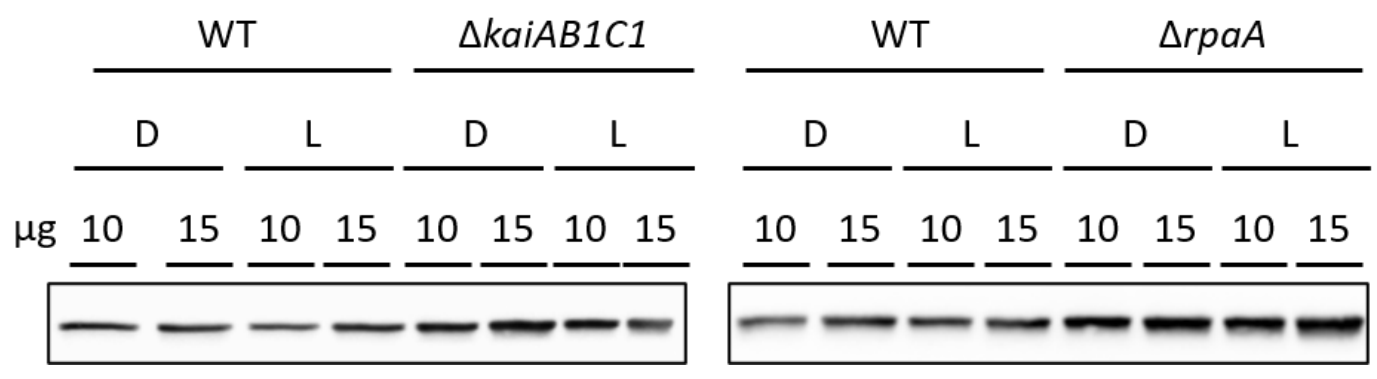

Figure 8. Increased RubisCO abundance in mutants compared to wild type (WT). Comparison of the RubisCO levels via immunoblot analysis using anti-RbcL. 10 or $15 \mu \mathrm{g}$ of crude cell extract were used for SDS-PAGE. The samples were collected $5.5 \mathrm{~h}$ after the transition to D or L as shown in Figure 4A. 


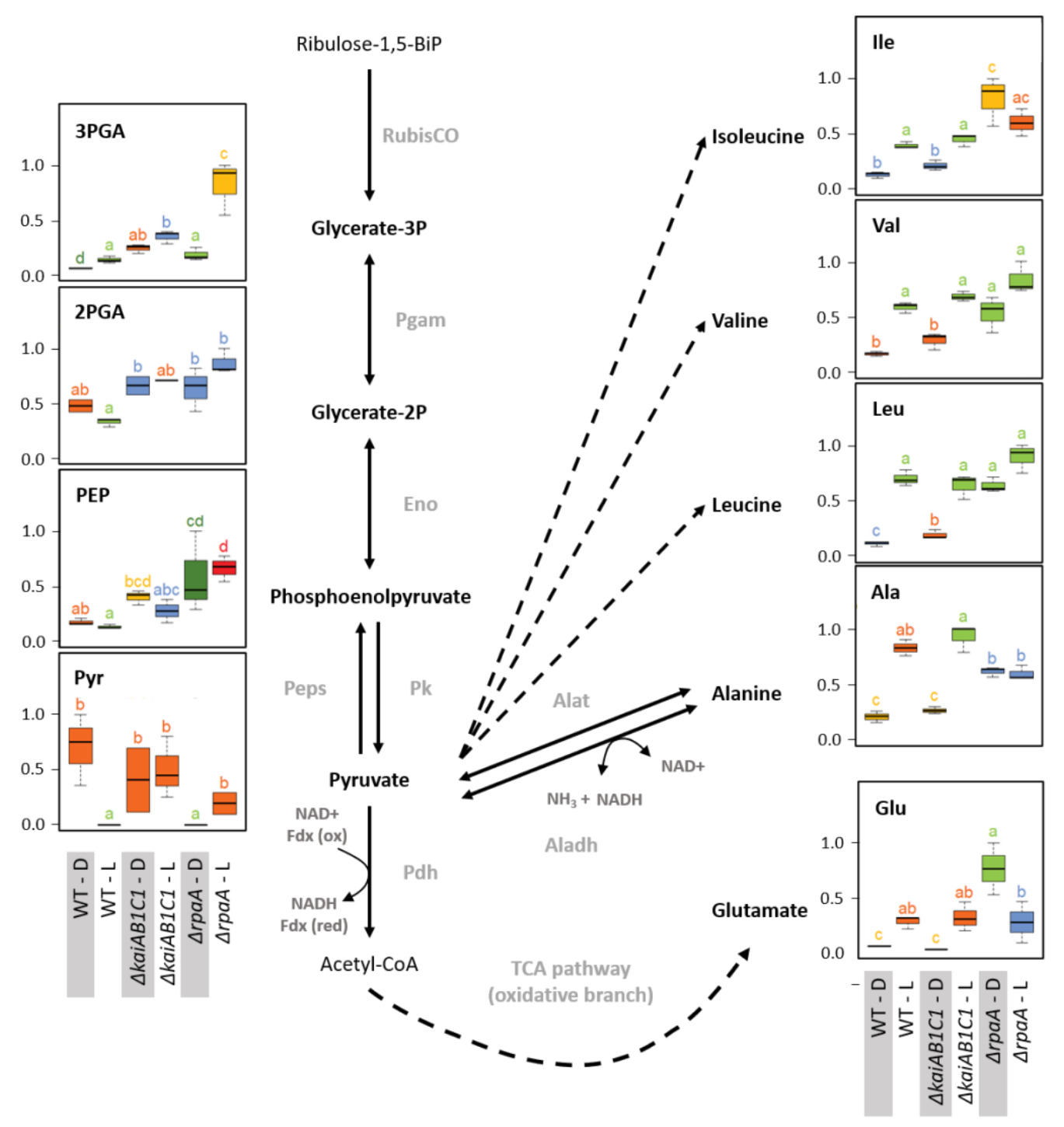

Figure 9. Lower glycolysis, glutamate, and the pyruvate family of amino acids. Box plots showing the maximum normalized relative changes in the metabolite concentrations and Tukey test results $(P$ $<0.05, \mathrm{n}=3$ ). Metabolite levels that significantly differ are indicated by lowercase letters and color coding. Note the subtle changes in the $\triangle$ kaiABlCl mutant, while the $\triangle r p a A$ mutant exhibited significantly increased pyruvate family amino acids, 3PGA, PEP and glutamate in D. These changes are accompanied by pyruvate depletion in the $\mathrm{D}$ phase relative to the wild type. In the L, 3PGA accumulates in the $\triangle k a i A B 1 C 1$ mutant relative to the wild type. In the $\triangle r p a A$ mutant, 3PGA accumulates to even higher levels, and the 2PGA and PEP increases become significant. Dashed arrows indicate multiple reaction steps. Relevant enzymes and complexes include (gray) ribulose-1,5bisphosphate carboxylase-oxygenase (RubisCO), phosphoglycerate mutase (Pgam), enolase (Eno), phosphoenolpyruvate synthase (Peps), pyruvate kinase $(\mathrm{Pk})$, pyruvate dehydrogenase (Pdh), alanine dehydrogenase (Aladh), and alanine transaminase (Alat). 


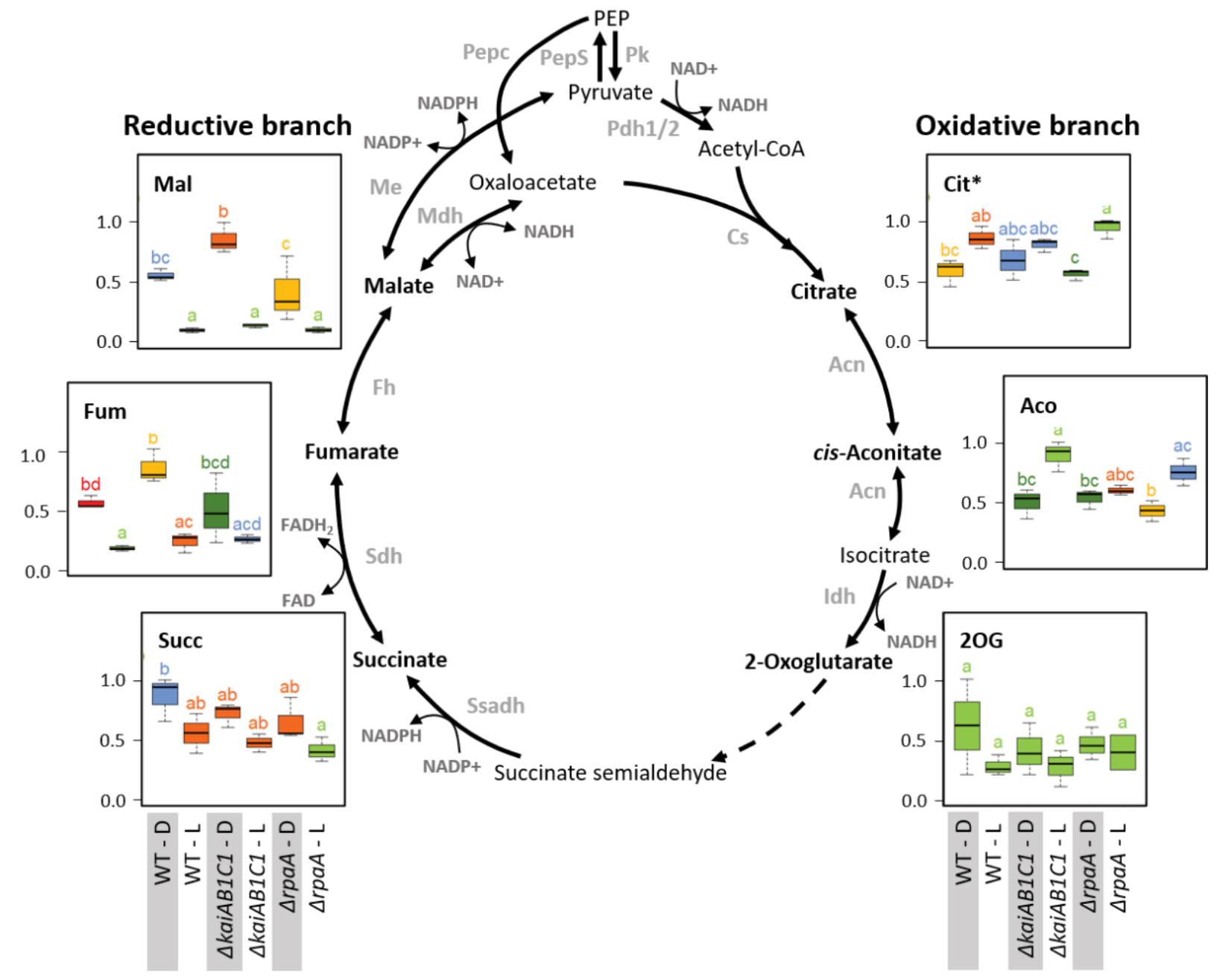

Figure 10. Tricarboxylic acid pathways. Box plots showing the maximum normalized relative changes in the metabolite concentrations and Tukey test results $(P<0.05, \mathrm{n}=3)$. Metabolite levels that significantly differ are indicated by lowercase letters and color coding. Note the inverse accumulation of metabolites from the oxidative and reductive branches of the TCA pathways. The concentrations of the intermediates of the TCA reactions were largely unchanged in the $\triangle$ kaiABlCl and $\triangle r p a A$ cells. Dashed arrows indicate multiple reaction steps. Relevant enzymes and complexes included (gray) phosphoenolpyruvate synthase (PepS), pyruvate kinase $(\mathrm{Pk})$, pyruvate dehydrogenase (Pdh), phosphoenolpyruvate carboxylase (Pepc), citrate synthase (Cs), aconitase (Acn), isocitrate dehydrogenase (Idh), succinate semialdehyde dehydrogenase (Ssadh), succinate dehydrogenase (Sdh), fumarase (Fh), malate dehydrogenase (Mdh), and malic enzyme (Me). 


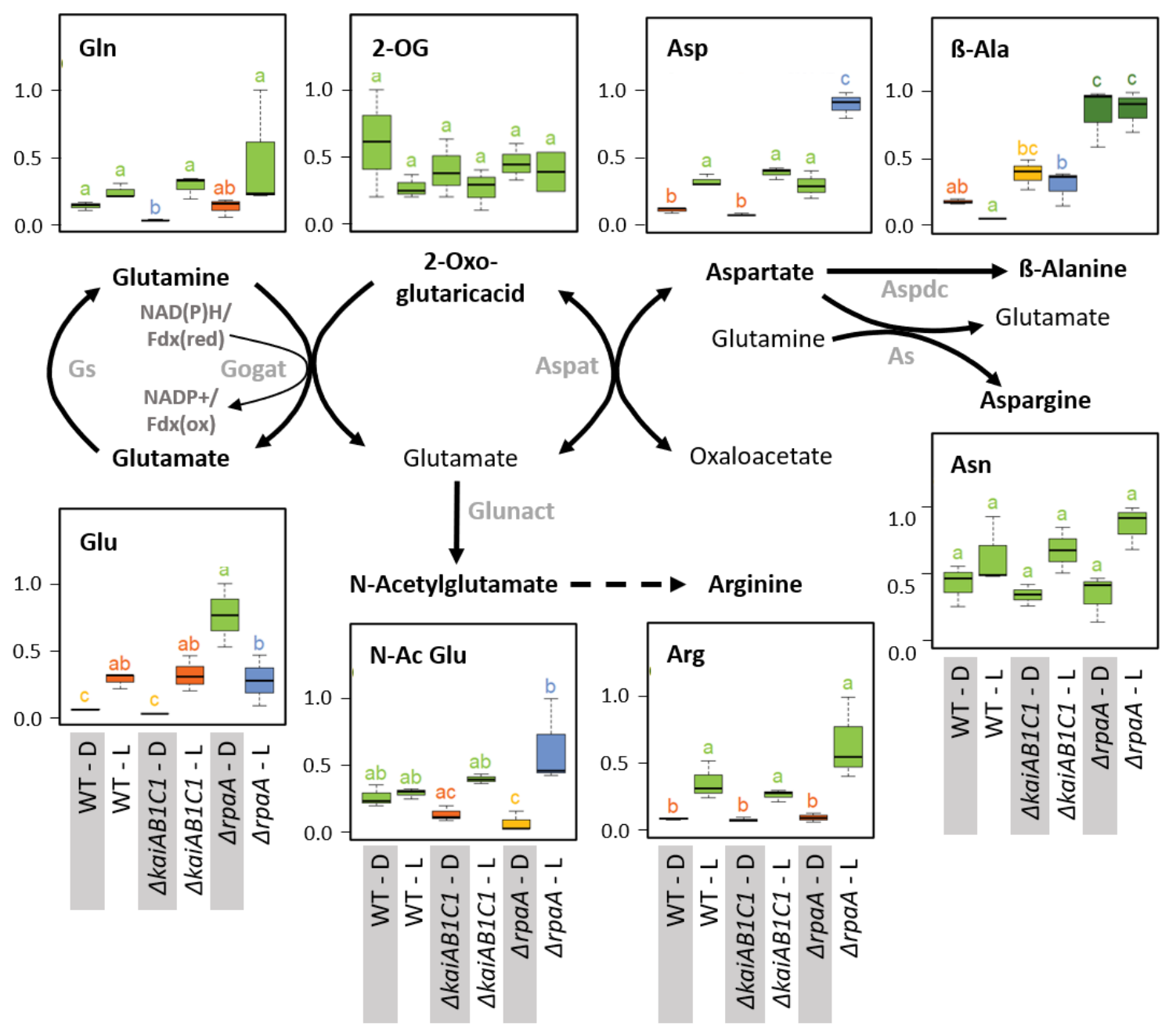

Figure 11. Nitrogen assimilation and associated amino acid biosynthesis. Box plots showing the maximum normalized relative changes in the metabolite concentrations and Tukey test results $(P<$ $0.05, \mathrm{n}=3$ ). Metabolite levels that significantly differ are indicated by lowercase letters and color coding. Note the increase in aspartate and the D accumulation of glutamate (Figure 11) in the $\Delta r p a A$ mutant. Relevant enzymes and complexes include (gray) glutamine synthetase (Gs), glutamine 2oxoglutarate aminotransferase (Gogat), aspartate aminotransferase (Aspat), asparagine synthetase (As), aspartate decarboxylase (Aspdc), and glutamate N-acetyltransferase (Glunact). 